\title{
THE RELATION BETWEEN TREE SIZE COMPLEXITY AND PROBABILITY FOR BOOLEAN FUNCTIONS GENERATED BY UNIFORM RANDOM TREES*
}

\author{
VERONIKA DAXNER, ANTOINE GENITRINI ${ }^{\dagger}$, BERNHARD GITTENBERGER $^{\ddagger}$, AND CÉCILE MAILLER $^{\mathcal{P}}$
}

\begin{abstract}
We consider a probability distribution on the set of Boolean functions in $n$ variables which is induced by random Boolean expressions. Such an expression is a random rooted plane tree where the internal vertices are labelled with connectives AND or OR and the leaves are labelled with variables or negated variables. We study the limiting distribution when the tree size tends to infinity and derive a relation between the tree size complexity and the probability of a function. This is done by first expressing trees representing a particular function as expansions of minimal trees representing this function and then computing the probabilities by means of combinatorial counting arguments relying on generating functions and singularity analysis.
\end{abstract}

Boolean functions; Probability distribution; Random Boolean formulas; Tree size complexity; Formula size complexity; Analytic combinatorics.

\section{INTRODUCTION}

A Boolean function of $n$ variables is a mapping from $\left\{\right.$ True, False ${ }^{n}$ onto $\{$ True, False $\}$. The interest in these objects dates back to the 1940ies when Riordan and Shannon [28, 30] discovered the so-called Shannon effect: the uniformly random $n$ variables Boolean function has asymptotically almost surely exponential complexity when $n$ goes to infinity. Since then numerous papers have been devoted to developing a better understanding of various aspects of Boolean functions. Concerning random Boolean functions and the Shannon effect, further investigations were carried out by Lupanov [24, 25] and a proof based on simple combinatorial counting arguments is presented in Flajolet and Sedgewick's book [8]. All these results concern the uniform probability distribution on the set of Boolean functions in $n$ variables.

In the last two decades people became interested in non-uniform distributions. A natural way to pick a Boolean function at random is to pick a Boolean formula at random and look at the function it represents. It is convenient to see Boolean formulas as rooted plane trees whose internal nodes are labelled by logical connectives like $\wedge, \vee$ or $\Longrightarrow$ (being respectively the conjunction, disjunction and implication operators) and whose leaves are labelled by (possibly negated) variables $\left\{x_{1}, \ldots, x_{n}\right\}$ (where $n$ is a fixed integer).

The first efforts in generating a random Boolean function via a random Boolean formula/tree go back to Paris et al. [26] and Lefmann and Savický [23] where the authors pick a tree uniformly at random among all binary and/or (meaning that only the connectives $\wedge$ and $\vee$ are allowed) trees having $m$ leaves. Lefmann and Savický [23] showed the existence of a limiting distribution when the size $m$ of the tree (i.e. its number of leaves) tends to infinity and also bounded the probability of a Boolean function in terms of its complexity, the complexity of a Boolean function being the size of the smallest trees representing it. The existence of a limit distribution when $m$ goes to infinity was shown independently for non binary trees by Woods [32. Woods' method is basically a special case of a more general result, the Drmota-Lalley-Woods theorem originating in the works [4, 22, 32. See Flajolet and Sedgewick [8] for an easy accessible formulation, Drmota [5] for a detailed discussion, and Fournier et al. [11] for an application in the context of Boolean formulas. The bounds of Lefmann and Savický were later refined in Chauvin et al. [3] by Analytic Combinatorics' methods. A survey of this topic was written by Gardy [12.

Different but somehow related problems in the framework of balanced trees have been pursued by Valiant [31] who wanted to generate a particular Boolean function with high probability. His results were extended in Boppana [1] and Gupta and Mahajan [18. The existence of a limit distribution for balanced and/or trees can be found in

*) This research was partially supported by the P.H.C. AMADEUS project 29281NE, the ÖAD project F03/2013 and the FWF grant SFB F50-03.

† Sorbonne Universités, UPMC Univ Paris 06, CNRS, LIP6 UMR 7606, 4 place Jussieu 75005 Paris. Antoine.Genitrini@lip6.fr.

$\ddagger$ Technische Universität Wien, Wiedner Hauptstrasse 8-10/104, A-1040 Wien, Austria. gittenberger@dmg.tuwien.ac.at

$\mathcal{P}$ Department of Mathematical Sciences, University of Bath, BA2 7AY Bath, UK. c.mailler@bath.ac.uk 
Fournier, Gardy and Genitrini [9]. The influence of different connectives was studied in Savický [29] and Brodsky and Pippenger 2, but under different distributions.

The study of the relation between the probability of a given Boolean function and its complexity under models similar to the Lefmann-Savický's model was resumed recently by Kozik 21] (and by Fournier et al. [10, 11] for the implicational model) who was able to obtain asymptotic equivalents for the probability of a fixed Boolean function $f$. More precisely, they prove that if $p_{n, m}(f)$ is the probability that a uniform binary and/or tree of size $m$ (and on $n$ variables) calculates $f$, then

$$
\lim _{m \rightarrow+\infty} p_{n, m}(f)=: p_{n}(f)=\Theta\left(\frac{1}{n^{\ell(f)+1}}\right), \text { when } n \rightarrow+\infty
$$

where $\ell(f)$ is the complexity of the Boolean function $f$.

The drawback of the models based on plane binary trees is that basic algebraic properties like commutativity and associativity of the connectives are not incorporated into the model. The papers [15, 14] extend the binary plane models to more general tree classes. For instance, due to the associativity of the connectives $\wedge$ and $\vee$, it is very natural to consider non binary trees, but in that case there is no justification why the size of an and/or tree should be its number of leaves (as considered in [15]) and not its number of internal nodes or its total number of nodes. And since the complexity of a Boolean function is defined as the size of the smallest trees calculating this function, changing the notion of size changes the notion of complexity.

In computer science, the formula size complexity (often called formula complexity or only complexity) is an important quantity in the investigation of Boolean functions. When looking at the tree representation of a Boolean formula, the formula size is the number of leaves of the tree. It is the size notion and thus the complexity notion used in the and/or trees literature mentioned above [26, 23, 3, 21]. This notion of size is natural in the binary connectives context (i.e. with gates of fanin 2) because, in that context, the number of connectives is the number of leaves shifted by 1 . However, when one turns to Boolean circuits, and consequently to (computational) complexity theory questions (see e.g. Graham, Grötschel and Lovász [17, Chapter 40]), the natural notion of size in this context is the number of connectives (or gates).

In this article, we consider non binary and/or trees. The leaves are not shared like in circuits, and thus both their number and the number of connectives are important when defining the size of a Boolean function. Moreover, from a computer science point of view, the size of the storage is a function of the number of all vertices. We thus define the tree size of a Boolean formula as the total number of nodes of its tree representation. The complexity of Boolean functions associated to this new notion of size is called the tree size complexity, as opposed to the formula size complexity used in the literature.

In this article, we show that the typical uniform and/or tree of size $m$ changes drastically when changing the size notion. This comes from the two successive limits taken in our set-up and in the literature: in Equation (1), the size of the trees first goes to infinity and then, we let the number $n$ of variables labelling the trees go to infinity. The typical and/or tree of size $m$ in the tree size model depends on the number of variables $n$ : the larger $n$ is, the more likely it is for the typical tree to have many leaves, whereas in the formula size model, the shape of the typical tree of size $m$ does not depend on $n$. This difference between the two models is the reason why we need to develop a whole new approach in this article since this difference of typical shapes make the proofs developed in the formula size literature collapse.

However, it eventually turns out that the two distributions induced on the set of Boolean functions by the tree size model and the formula size model very well fall under the same paradigms. As in the formula size model (see Equation (1)), we can prove that there is a strong relation between the (tree size) complexity and the probability of a function. More precisely, if we pick uniformly at random an and/or tree of tree size $m$ (labelled on $n$ variables) and denote by $\mathbb{P}_{m, n}(f)$ the probability that this tree represents the Boolean function $f$, then we prove in this article that

$$
\lim _{m \rightarrow+\infty} \mathbb{P}_{m, n}(f)=: \mathbb{P}_{n}(f)=\Theta\left(\frac{1}{n^{L(f)}}\right),
$$

where $L(f)$ is the tree size complexity. This result is very close to Equation (1), but the difference in the exponent of $n$ actually makes a clear difference, namely that in our model, the asymptotic probability of the two constant functions True and False (which have complexity 0) is of constant order while it was tending to zero with $n$ in the formula size model. This immediately implies that the tree size model does not exhibit the Shannon effect, whereas proving that the formula size model does not exhibit the Shannon effect is proved but far from trivial (see [13, 14, 16]). 


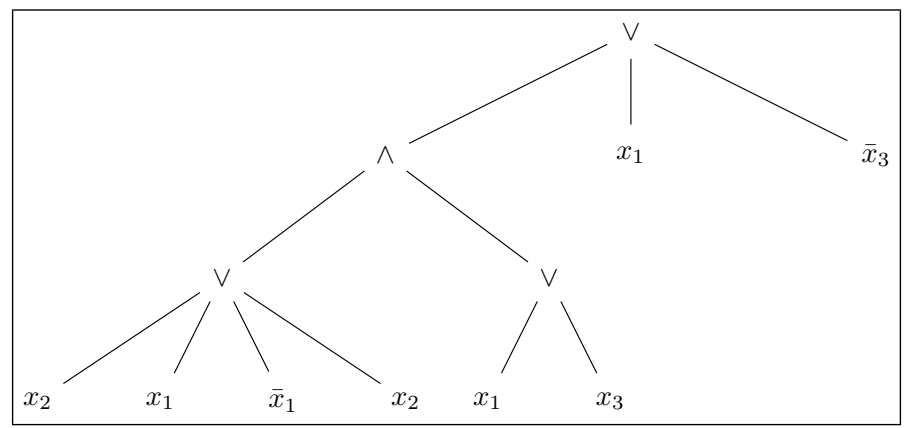

FiguRE 1. An associative tree which represents the constant function True.

The present paper is organized as follows: In the next section we introduce the model and present our main result. In Section 3 we introduce the combinatorial setting (generating functions for the basic tree classes) and briefly discusses the existence of the limit distribution $\mathbb{P}_{n}$ (see Equation (2)). In Section 4 is devoted to proving key properties of our model: In particular, we investigate in this part the typical shape of the random uniform and/or tree of size $m$, when $m$ and then $n$ tend to infinity. This section shows how different the typical tree is our model and the typical tree in the formula size model are different. In Section 5 we study a particular subfamily of trees which will be used as an auxiliary structure in Sections 6 and 9 In Section 6, we prove that the probability of the two constant functions is of constant order when $n$ tends to infinity, meaning that we prove Equation (2) in the special cases $f=$ True and $f=$ False. A fixed Boolean function divides the Boolean lattice into larger and smaller functions (as well as non-comparable functions). This subdivision is quantified in Section 7 and the result will help us to estimate the limiting probability of literal functions, the simplest non-constant functions, in Section 8 , We finally have all ingredients needed to prove our main result (i.e. Equation (2), which we do in Section 9 .

\section{Model And Results}

Definition 2.1. An associative tree is a rooted plane tree whose nodes have arity in $\mathbb{N} \backslash\{1\}$, and such that each internal node is labelled by a connector AND (denoted by $\wedge$ in the following) or by a connector OR (denoted by $\vee$ in the following) such that two identical connectives cannot be neighbours (trees are stratified), and where each leaf is labelled by a literal taken from $\left\{x_{1}, \bar{x}_{1}, \ldots, x_{n}, \bar{x}_{n}\right\}$ (see Figure 1 for an example).

By interpreting $\bar{x}_{i}$ as the negation of $x_{i}$, every such tree represents a Boolean formula (or expression) and therefore calculates a Boolean function of $n$ variables. We denote by $\mathcal{F}_{n}$ the set of such Boolean functions.

Definition 2.2. The size $|t|$ of an associative tree $t$ is the number of its nodes (internal nodes and leaves). We denote by $\mathcal{A}_{m, n}$ the set of associative trees of size $m$, and by $A_{m, n}$ the cardinality of this set. Let $f \in \mathcal{F}_{n}$ be a Boolean function, its complexity is

- 0 if $f$ is the function True : $\left(x_{1}, \ldots, x_{n}\right) \mapsto$ True or False $:\left(x_{1}, \ldots, x_{n}\right) \mapsto$ False;

- 2 if $f$ is a literal function, i.e. there exist $x_{i} \in\left\{x_{1}, \ldots, x_{n}\right\}$ such that $f:\left(x_{1}, \ldots, x_{n}\right) \mapsto x_{i}$ or $f$ : $\left(x_{1}, \ldots, x_{n}\right) \mapsto \bar{x}_{i}$

- the size of the smallest trees computing $f$ if $f$ is neither a literal function nor a constant function. These trees of size $L(f)$ computing $f$ are called minimal trees of $f$ and their set is denoted by $\mathcal{M}_{f}$.

Example: The tree pictured in Figure 1 has size 11. The function $\left(x_{1}, \ldots, x_{n}\right) \mapsto x_{1}$ XOR $x_{2}=\left(\bar{x}_{1} \wedge x_{2}\right) \vee\left(x_{1} \wedge \bar{x}_{2}\right)$ has complexity 7 .

As already mentioned in the introduction, this notion of size differs from the notion used in the literature about and/or tree, where the size of a tree is the number of its leaves. Changing the notion of size also changes the notion of complexity of Boolean functions. In all discussions, we will call the complexity defined in Definition 2.2 the tree size complexity, as opposed to the formula size complexity of the literature. As discussed in the introduction, this alternative definition is at least as natural and it is an interesting question to know how this change of definition will impact the distribution induced on the set of Boolean functions by the uniform distribution on the set of and/or trees of size $m$ and labelled on $n$ variables. 
Remark: The literal functions will be treated separately in the whole paper. But they could in fact be treated as any other non-constant function by considering that its two minimal trees are the tree rooted by an $\wedge$ with a single child labelled by $x_{i}$ and the tree rooted by an $\vee$ with a single child labelled by $x_{i}$, even if those two trees are not associative trees according to Definition 2.1

Definition 2.3. For all Boolean function $f$, we denote by $\mathbb{P}_{m, n}(f)$ the proportion of trees calculating $f$ among all trees of size $m$.

The aim of the present paper is to prove the following result, which sums up the asymptotic behaviour of this distribution $\mathbb{P}_{m, n}$ when the size $m$ of the considered tree tends to infinity.

Theorem 2.4. For all Boolean function $f$, the following limit exists and is positive

$$
\mathbb{P}_{n}(f)=\lim _{m \rightarrow \infty} \mathbb{P}_{m, n}(f) .
$$

Moreover,

- There exist two constants $\alpha$ and $\beta$ such that, for all integer $n$,

$$
0<\alpha \leq \mathbb{P}_{n}(\text { True })=\mathbb{P}_{n}(\text { False }) \leq \beta<\frac{1}{2} .
$$

- If $f$ is a literal function, then

$$
\mathbb{P}_{n}(f)=\Theta\left(\frac{1}{n^{2}}\right) \text { when } n \rightarrow+\infty .
$$

- For all Boolean function $f$ such that $L(f) \geq 3$,

$$
\mathbb{P}_{n}(f)=\Theta\left(\frac{1}{n^{L(f)}}\right) \text { when } n \rightarrow+\infty .
$$

Remark: Thanks to the ad hoc definition of the complexity ( $c f$. Definition 2.2), the above theorem can be expressed as: For all Boolean function $f$,

$$
\mathbb{P}_{n}(f)=\Theta\left(\frac{1}{n^{L(f)}}\right), \text { as } n \text { tends to infinity. }
$$

As already mentioned in the introduction, our main result shows that changing the notion of size/complexity does not affect the behaviour of the induced distribution on Boolean functions: the main result above is very similar to Equation (1), valid in the formula size complexity. Therefore, one could expect that a slight generalisation of the proofs developed in the literature for the formula size complexity might be enough to prove the above main result. It might come as a surprise that generalising the formula size complexity proofs collapse in our context due to the difference of the shapes of a typical uniform tree of size $m$ in both models - a glimpse of these differences will be given in Section 4 but roughly speaking, the number of leaves attached at the root is of order $\sqrt{n}$ while it did not depend on $n$ in the literature model. Our main contribution is thus the development of a whole new approach to prove our main result.

\section{Existence of the Limiting Distribution $\mathbb{P}_{n}$}

In this section, we prove the existence of the limiting distribution $\mathbb{P}_{n}$ on the set $\mathcal{F}_{n}$, which correspond to the first assertion of Theorem 2.4 In the whole paper, we deeply use generating functions, singularity analysis and the symbolic method. We refer the reader to [8] for a comprehensive introduction to this domain.

An associative tree can be formally described by a grammar: If $\hat{\mathcal{A}}$ (resp. $\breve{\mathcal{A}}$ ) denotes the set of all associative trees rooted by an $\wedge$-connective (resp. an $\vee$-connective), $\mathcal{L}$ the set of literals and $\mathcal{C}=\{\wedge\}$, then $\hat{\mathcal{A}}=\mathcal{L}+\mathcal{C} \times \operatorname{seq}_{\geq 2}(\check{\mathcal{A}})$. Let us denote by $\hat{A}(z)$ (resp. $\check{A}(z)$ ) the generating function of associative trees rooted by an $\wedge$-connective (resp. an $\checkmark$-connective). The grammar can be translated into the functional equation

$$
\hat{A}(z)=2 n z+z \cdot \frac{\check{A}(z)^{2}}{1-\check{A}(z)} .
$$


Since, by symmetry, $\check{A}(z)=\hat{A}(z)$, we get

$$
\hat{A}(z)=\frac{2 n z+1-\sqrt{\left(4 n^{2}-8 n\right) z^{2}-4 n z+1}}{2(z+1)} .
$$

If we denote by $A(z)=\sum_{m \geq 0} A_{m, n} z^{m}$ the generating function of all associative trees, then

$$
A(z)=2 \hat{A}(z)-2 n z .
$$

Moreover, let $\hat{A}_{f}(z)$ (resp. $\left.\check{A}_{f}(z)\right)$ denote the generating function of associative trees rooted by an $\wedge($ resp. an $\vee$ ) connective and computing the Boolean function $f$. Using the symbolic method, we get

$$
\begin{aligned}
& \hat{A}_{f}(z)=z \cdot \mathbb{1}_{f \mathrm{lit}}+z \cdot \sum_{\ell \geq 2} \sum_{g_{1} \wedge \cdots \wedge g_{\ell}=f} \check{A}_{g_{1}}(z) \ldots \check{A}_{g_{\ell}}(z) \\
& \check{A}_{f}(z)=z \cdot \mathbb{1}_{f \mathrm{lit}}+z \cdot \sum_{\ell \geq 2} \sum_{g_{1} \vee \cdots \vee g_{\ell}=f} \hat{A}_{g_{1}}(z) \ldots \hat{A}_{g_{\ell}}(z) .
\end{aligned}
$$

We thus have a system of functionnal equations to which we will apply the Drmota-Lalley-Woods. Let us check the hypothesis of this theorem: We refer the reader to [7, page 489] for a precise statement of the Drmota-LalleyWoods theorem for polynomial systems. A more general form can be found in the original work [4] and in [5] Section 2.2.5]. The above system is non-linear, it has nonnegative coefficients and it satisfies a Lipschitz condition. It is irreducible, because for every function $f$ we have $f \wedge$ True $\equiv f$ and $f \vee$ True $\equiv$ True. Finally, note that a tree having a $\vee$-root and two subtrees, one being only one leaf labelled by $x_{1}$ and the other one a tree of arbitrary size but with all leaves labelled by $\bar{x}_{1}$, is a tautology. Thus, for all $m \geq 3$, there exists a tree of size $m$ calculating the constant function True and hence $\check{A}_{\text {True }}(z)$ is a-periodic. This is enough to imply that all the functions $\hat{A}_{f}(z)$ and $\check{A}_{f}(z)$ are also a-periodic. Thus we have shown that all hypotheses of the Drmota-Lalley-Woods are true.

We can therefore infer that all the generating functions $\hat{A}_{f}(z), \check{A}_{f}(z)$ and $A(z)$ have the same unique singularity $\rho>0$ on their (common) circle of convergence, it is a square-root singularity and $\hat{A}_{f}(z), \check{A}_{f}(z)$ as well as $A(z)$ admit a singular expansion of the same type (Puiseux expansion in terms of powers of $\sqrt{\rho-z}$ at $\rho$. Applying a transfer lemma (see [7]) to $A(z), \hat{A}_{f}(z)$, and $\check{A}_{f}(z)$ we can conclude that the limiting distribution $\mathbb{P}_{n}(f)=\lim _{m \rightarrow \infty} \frac{\left[z^{m}\right]\left(\hat{A}_{f}(z)+\check{A}_{f}(z)\right.}{\left[z^{m}\right] A(z)}$ exists for all $f$, proving the first statement of Theorem 2.4

Knowing the generating function of $\wedge$-rooted associative trees (given in Equation (3)), we can state the following proposition that will be widely used later on.

Proposition 3.1. The singularity $\rho$ of $\hat{A}(z)$ satisfies, as $n \rightarrow \infty$,

$$
\rho=\frac{1}{2} \cdot \frac{1}{n+\sqrt{2 n}}=\frac{1}{2 n}-\frac{1}{n \sqrt{2 n}}+\mathcal{O}\left(\frac{1}{n^{2}}\right)
$$

in particular, for all large enough $n$, we have $\frac{1}{2 n}-\frac{1}{2 n \sqrt{n}} \leq \rho<\frac{1}{2 n}$. Moreover,

$$
A(\rho)=1-\frac{1}{n}+\mathcal{O}\left(\frac{1}{n \sqrt{n}}\right) \quad \text { and } \quad \hat{A}(\rho)=1-\frac{1}{\sqrt{2 n}}+\mathcal{O}\left(\frac{1}{n}\right) .
$$

Finally, if $\hat{B}(z)$ denotes the generating function of $\wedge$-rooted associative trees, then $\hat{B}(z)=\hat{A}(z)-2 n z$. For all integer $n$,

$$
\hat{B}(\rho)<\frac{1}{\sqrt{2 n}} \quad \text { and } \quad \hat{B}(\rho)=\frac{1}{\sqrt{2 n}}+\mathcal{O}\left(\frac{1}{n}\right) \text { when } n \rightarrow+\infty .
$$

\section{Miscellaneous properties of the model.}

In this section, we prove several propositions that lead to a better understanding of the model and that will be useful throughout the paper. We prove among other results that the expected number of leaves on the first level of a large associative tree behaves like $\sqrt{n}$, and that there are few trees with no leaves on the first level. All these results are really surprising in view of the formula size models of the literature where the number of leaves attached to the root of a typical tree of large size does not depend on the number $n$ of variables. Therefore, in the present model, it is quite likely to have a literal and its negation appearing as labels of two leaves attached to the root, making the whole tree calculate one of the two constant functions, whereas this configuration is very unlikely in the formula size model (see [15]). 
First of all, let us define the limiting ratio of a family:

Definition 4.1. Let $\mathcal{T}$ be a family of associative trees and $T_{m}$ be the number of trees of size $m$ in this family. The limiting ratio of $\mathcal{T}$, if it exists, is defined (and denoted) by

$$
\mu_{n}(\mathcal{T})=\lim _{m \rightarrow \infty} \frac{T_{m}}{A_{m, n}}
$$

The following standard result will be extensively used in the following:

Lemma 4.2. Let $T(z)$ be the generating function of a family $\mathcal{T}$ of associative trees. Assume that $\rho$ (cf. Proposition (3.1) is the unique singularity of $T(z)$ on its circle of convergence and that this singularity is of square-root type, i.e. $T(z)$ admits a Puiseux expansion into powers of $\sqrt{\rho-z}$ at $\rho$. Then

$$
\mu_{n}(\mathcal{T})=\lim _{z \rightarrow \rho} \frac{T^{\prime}(z)}{A^{\prime}(z)}
$$

where $z$ must move towards $\rho$ in such a way that $\arg (z-\rho) \neq 0$ and inside the domain of analyticity of $T(z)$ and $A(z)$.

Proof. This is an immediate consequence of the fact that the asymptotic expansion of the derivative of an analytic function is the derivative of the asymptotic expansion of the original function.

Proposition 4.3. The limiting ratio of trees with no leaf on the first level is given by

$$
\mu_{n}\left(\mathcal{A}^{(0)}\right)=\frac{1}{n \sqrt{2 n}}+\mathcal{O}\left(\frac{1}{n^{2}}\right) \text {, as } n \rightarrow \infty .
$$

Proof. The generating function of trees with no leaf on the first level is given by

$$
A^{(0)}(z)=2 z \frac{\hat{B}(z)^{2}}{1-\hat{B}(z)} .
$$

The limiting ratio of such trees is thus given by

$$
\begin{aligned}
\mu_{n}\left(\mathcal{A}^{(0)}\right) & =\lim _{m \rightarrow \infty} \frac{\left[z^{m}\right] A^{(0)}(z)}{\left[z^{m}\right] A(z)}=\lim _{z \rightarrow \rho} \frac{A^{\prime(0)}(z)}{A^{\prime}(z)} \\
& =2 \rho \frac{2 \hat{B}(\rho)}{1-\hat{B}(\rho)} \lim _{z \rightarrow \rho} \frac{\hat{B}^{\prime}(z)}{A^{\prime}(z)}+2 \rho \frac{\hat{B}(\rho)^{2}}{(1-\hat{B}(\rho))^{2}} \lim _{z \rightarrow \rho} \frac{\hat{B}^{\prime}(z)}{A^{\prime}(z)}+2 \frac{\hat{B}(\rho)^{2}}{1-\hat{B}(\rho)} \lim _{z \rightarrow \rho} \frac{1}{A^{\prime}(z)} .
\end{aligned}
$$

Observe that the third term of the sum is equal to zero since $A^{\prime}(z)$ tends to infinity when $z$ tends to $\rho$. This observation will be used in the whole paper and in the following, such terms will be omitted without mentioning. Note also that $\lim _{z \rightarrow \rho} \hat{B}^{\prime}(z) / A^{\prime}(z)=1 / 2$. Finally, use the asymptotics given in Proposition 3.1 and get

$$
\mu_{n}\left(\mathcal{A}^{(0)}\right)=\frac{1}{n \sqrt{2 n}}+\mathcal{O}\left(\frac{1}{n^{2}}\right) \text { as } n \rightarrow \infty \text {. }
$$

Proposition 4.4. Let $\Gamma$ be a subset of $\gamma$ literals of $\left\{x_{1}, \bar{x}_{1}, \ldots, x_{n}, \bar{x}_{n}\right\}$. The limiting ratio of the set of all associative trees with at least one leaf on the first level labelled by a literal from $\Gamma$ is given by

$$
\mu_{n}\left(\mathcal{A}_{\Gamma}\right)=\gamma \sqrt{\frac{2}{n}}+\mathcal{O}\left(\frac{1}{n}\right) \text {, when } n \text { tends to infinity. }
$$

Proof. Let $A_{\Gamma}(z)$ be the generating function of associative trees with at least one leaf one the first level labelled by a literal from $\Gamma$ :

$$
A_{\Gamma}(z)=\frac{2 \gamma z^{2}}{(1-(\hat{A}(z)-\gamma z))(1-\hat{A}(z))}-2 \gamma z^{2}
$$

because a tree in $\mathcal{A}_{\Gamma}$ has a root labelled by $\wedge$ or $\vee$ (which gives a factor $2 z$ ), a first sequence of subtrees which are not a leaf labelled by a literal from $\Gamma$ (which gives the factor $\left.\frac{1}{1-(\hat{A}(z)-\gamma z)}\right)$, then a leaf labelled by literal from $\Gamma$ (factor $\gamma z$ ) and a sequence of arbitrary trees. Since sequences may be empty, this construction also generates trees consisting of only two nodes. These have to be subtracted due to the vertex degree constraints in associative trees. In view of Lemma 4.2 , we know that

$$
\mu_{n}\left(\mathcal{A}_{\Gamma}\right)=\lim _{m \rightarrow \infty} \frac{\left[z^{m}\right] A_{\Gamma}(z)}{\left[z^{m}\right] A(z)}=\lim _{z \rightarrow \rho} \frac{A_{\Gamma}^{\prime}(z)}{A^{\prime}(z)}=\gamma \sqrt{\frac{2}{n}}+\mathcal{O}\left(\frac{1}{n}\right) .
$$


Proposition 4.5. Let $X_{m, n}$ be the number of leaves in the first level of an associative tree of size $m$. Then $\lim _{m \rightarrow \infty} \mathbb{E}\left(X_{m, n}\right) \sim 2 \sqrt{2 n}$, as $n \rightarrow \infty$.

Proof. Let us consider the bivariate generating function where $z$ marks the nodes and $u$ the leaves on the first level. We have the following equation:

$$
A(z, u)=2 z \frac{(\hat{B}(z)+2 n z u)^{2}}{1-(\hat{B}(z)+2 n z u)} .
$$

Therefore,

$$
\frac{\partial}{\partial u} A(z, u)_{\mid} u=1=8 n z^{2} \frac{\hat{A}(z)}{1-\hat{A}(z)}+4 n z^{2} \frac{\hat{A}(z)^{2}}{(1-\hat{A}(z))^{2}} .
$$

In view of Lemma 4.2 the expected number of nodes in the first level is given by

$$
\begin{aligned}
\lim _{m \rightarrow \infty} \frac{\left[z^{m}\right] \frac{\partial}{\partial u} A(z, u)_{\mid} u=1}{\left[z^{m}\right] A(z)} & =\lim _{z \rightarrow \rho} \frac{\frac{d}{d z}\left(\frac{\partial}{\partial u} A(z, u)_{\mid} u=1\right)}{\frac{d}{d z} A(z)} \\
& =2 \sqrt{2 n}+\mathcal{O}(1),
\end{aligned}
$$

as $n \rightarrow \infty$.

Remark: Using a similar calculation but plugging $u=e^{i t / 2 \sqrt{2 n}}$ instead of $u=1$, we can obtain even the limiting distribution. Indeed, one easily shows that the distribution of the random variable $X_{n} / 2 \sqrt{2 n}$ converges to the $\Gamma(2,1 / 2)$ distributed, as $n \rightarrow \infty$, i.e., the distribution having density $4 x e^{-2 x} \mathbb{1}_{x \geq 0}$.

\section{A useful FAmily of trees}

In the following, we will need some information about a specific class of trees which will serve as an auxiliary construction for our further investigations. We start with the definition of this particular family of trees and then study its limiting ratio.

Definition 5.1. Let $k, r, \ell \geq 0$ be three integers, let $\Gamma=\left\{\gamma_{1}, \ldots, \gamma_{p}\right\}$ be a subset of literals (with no occurrence of both a variable and its negation). Let $\mathcal{M}_{k, \ell, r}^{\Gamma}$ be the family of $\vee$-rooted trees

- with exactly $k$ different literals $\alpha_{1}, \ldots, \alpha_{k}$ appearing as labels of leaves on the first level, such that both a variable and its negation cannot appear, and for all $i=1, \ldots, k$ and $j=1, \ldots, p$ we have $\alpha_{i} \neq \gamma_{j}$,

- with the root having exactly $\ell$ non-leaf subtrees,

- and with at least one non-leaf subtree chosen from the family $\mathcal{J}_{k, r}^{\Gamma}$ :

The family $\mathcal{J}_{k, r}^{\Gamma}$ contains all $\wedge$-rooted trees such that

- there are $r$ leaves in the first level carrying pairwise different labels $\beta_{1}, \ldots, \beta_{r}$ (with no occurrence of both a variable and its negation) which are different from $\alpha_{1}, \ldots, \alpha_{k}, \gamma_{1}, \ldots, \gamma_{p}$ as well as their negations;

- all the other leaves on the first level have labels from $\left\{\alpha_{1}, \ldots, \alpha_{k}, \gamma_{1}, \ldots, \gamma_{p}, \beta_{1} \ldots, \beta_{r}\right\}$ or their negations,

Lemma 5.2. Let $J(z)$ and $M(z)$ denote the generating functions associated with $\mathcal{J}_{k, r}^{\gamma}$ and $\mathcal{M}_{k, \ell, r}^{\Gamma}$, respectively. Then

$$
J(z)=\frac{z^{r+1}}{(1-(\hat{B}(z)+2(k+p) z)) \ldots(1-(\hat{B}(z)+2(k+p+r) z))}
$$

and

$$
\begin{aligned}
{\left[z^{m}\right] M(z) \leq } & \left(\begin{array}{c}
n-p \\
k+r
\end{array}\right)\left(\begin{array}{c}
k+r \\
k
\end{array}\right) \frac{2^{k+r} k ! r !}{(\ell-1) !} \\
& \times\left[z^{m-1}\right]\left(z^{k+\ell} \prod_{\nu=1}^{k} \frac{1}{1-\nu z}\right)^{(\ell)} \frac{z^{r+1}}{\prod_{\nu=0}^{r}(1-(\hat{B}(z)+(k+p+\nu) z))} \hat{B}(z)^{\ell-1},
\end{aligned}
$$

for all $m \geq 0$, where $f^{(\ell)}$ denotes the $\ell$ th derivative of $f$. 
Proof. The first equation is obvious from the definition of $\mathcal{J}_{k, r}^{\gamma}$. Since Equation (6) is an inequality and not an equality we can do the following reasoning without worrying about double-counting some trees. Let us follow the definition of $\mathcal{M}_{k, \ell, r}^{\Gamma}$ : First note that there are $\left(\begin{array}{c}n-p \\ k+r\end{array}\right)\left(\begin{array}{c}k+r \\ k\end{array}\right) 2^{k+r} r ! k$ ! ways to choose $\alpha_{1}, \ldots, \alpha_{k}$ and $\beta_{1}, \ldots, \beta_{r}$ (and their respective order) such that a variable and its negation cannot both be chosen. The leaves of the first level form a sequence of $\alpha_{1}$ followed by the first occurrence of $\alpha_{2}$, then a sequence of leaves with labels in $\left\{\alpha_{1}, \alpha_{2}\right\}$ followed by the first occurrence of $\alpha_{3}$, and so on. This corresponds to the generating functions $z^{k} \prod_{\nu=1}^{k} \frac{1}{1-\nu z}$ and if we choose the places of the $\ell$ non-leaf subtrees of the root between the leaves of the first level, then we have to apply the operator ${ }^{1} \frac{1}{\ell !} \frac{\partial^{\ell}}{\partial z^{\ell}}$. The generating function of the non-leaf subtree taken from $\mathcal{J}_{k, \ell, r}^{\Gamma}$ and its place among the non-leaf subtrees is $\ell J(z)$ and that of the other non-leaf subtrees is $\hat{B}(z)^{\ell-1}$. When we collect all these terms and take into account that multiple counting occurs in the cases where several non-leaf subtrees of the root are in $\mathcal{J}_{k, \ell, r}^{\Gamma}$, then we get

$$
\left[z^{m}\right] M(z) \leq\left[z^{m}\right] z\left(\begin{array}{c}
n-p \\
k+r
\end{array}\right)\left(\begin{array}{c}
k+r \\
k
\end{array}\right) 2^{k+r} k ! \frac{1}{\ell !}\left(z^{k+\ell} \prod_{\nu=1}^{k} \frac{1}{1-\nu z}\right)^{(\ell)} \ell J(z) \hat{B}(z)^{\ell-1},
$$

where the factor $z$ at the very beginning stands for the root. Finally, using Equation (5) to replace $J(z)$ in Equation (7) gives Equation (6) as desired.

By means of the previous lemma we can express the limiting ratio of the family $\mathcal{M}_{k, \ell, r}^{\Gamma}$ in the form

$$
\lim _{z \rightarrow \rho} \frac{M^{\prime}(z)}{A^{\prime}(z)}=\left(\begin{array}{c}
n-p \\
k+r
\end{array}\right)\left(\begin{array}{c}
k+r \\
k
\end{array}\right) 2^{k+r} \frac{2^{k+r} k ! r !}{(\ell-1) !}\left(\rho^{k+\ell} \prod_{\nu=1}^{k} \frac{1}{1-\nu \rho}\right)^{(\ell)} \rho^{r+2} \lim _{z \rightarrow \rho} \frac{K^{\prime}(z)}{A^{\prime}(z)}
$$

where $K(z)=\frac{\hat{B}(z)^{\ell-1}}{\prod_{\nu=0}^{r}(1-(\hat{B}(z)+(k+p+\nu) z))}$. The only term depending on $z$ is $K^{\prime}(z) / A^{\prime}(z)$. The next lemma analysis this term.

Lemma 5.3. Let $K(z)$ be as above. Moreover, assume that $k+p+r<n$ and $r=\mathcal{O}(\sqrt{n})$. Then

$$
\lim _{z \rightarrow \rho} \frac{K^{\prime}(z)}{A^{\prime}(z)}=\mathcal{O}\left(r 2^{r} \ell\left(\frac{1}{2 n}\right)^{\frac{\ell-1}{2}}\right),
$$

as $n \rightarrow \infty$.

Proof. We have, as $z \rightarrow \rho$,

$$
\frac{K^{\prime}(z)}{A^{\prime}(z)} \sim \frac{\hat{B}^{\prime}(z)}{A^{\prime}(z)} \frac{1}{\prod_{m=0}^{r}(1-\hat{B}(\rho)-(k+p+m) \rho)}\left[(\ell-1) \hat{B}(\rho)^{\ell-2}+\sum_{m=0}^{r} \frac{\hat{B}(\rho)^{\ell-1}}{1-\hat{B}(\rho)-(k+p+m) \rho}\right] .
$$

Moreover, we know that $\lim _{z \rightarrow \rho} \frac{\hat{B}^{\prime}(z)}{A^{\prime}(z)}=1 / 2$ and that $\hat{B}(\rho) \leq \frac{1}{\sqrt{2 n}}(c f$. Proposition 3.1). Hence

$$
\frac{1}{\prod_{m=0}^{r}(1-\hat{B}(\rho)-(k+p+m) \rho)} \leq\left(\frac{1}{2}\left(1-\frac{\sqrt{2}}{\sqrt{n}}\right)\right)^{-r}=\mathcal{O}\left(2^{r}\right)
$$

we get

$$
\frac{K^{\prime}(z)}{A^{\prime}(z)}=\mathcal{O}\left(r 2^{r} \ell\left(\frac{1}{2 n}\right)^{\frac{\ell-1}{2}}\right) .
$$

The term in brackets in Equation (9) consists of $\mathcal{O}(r)$ terms, each bounded by $\ell \hat{B}(\rho)^{\ell-1}$ which immediately yields the assertion.

Lemma 5.4. If $k=\Omega\left(n^{1 / 4}\right), \ell=\mathcal{O}\left(n^{1 / 8}\right)$ and $r \leq \ell$, then the limiting ratio of the family $\mathcal{M}_{k, \ell, r}^{\Gamma}$ satisfies $\mu_{n}\left(\mathcal{M}_{k, \ell, r}^{\Gamma}\right)=\mathcal{O}\left(\frac{1}{n^{3 / 2}}\right)$, as $n$ tends to infinity.

\footnotetext{
${ }^{1}$ Recall that $\left[z^{m}\right] \frac{\left(z^{\ell} f(z)\right)(\ell)}{\ell !}=\left(\begin{array}{c}m+\ell \\ \ell\end{array}\right) f_{m}$.
} 
Proof. Turning back to (8) we see that the last factor is covered by Lemma 5.3 In order to estimate the penultimate factore, let $f(z)=z^{k+\ell} \prod_{\nu=1}^{k} 1 /(1-\nu z)$ and consider a circle of radius $n^{-3 / 2}$ centered at $\rho$. Since $f(z)$ is a power series with non-negative coefficients, Cauchy's estimate gives

$$
\left|\frac{f^{(\ell)}(\rho)}{\ell !}\right| \leq f\left(\rho+\frac{1}{n^{3 / 2}}\right) n^{3 \ell / 2}
$$

Now, performing the substitution $z=\rho+\frac{1}{n^{3 / 2}}$ into $f(z)$ on the right-hand side, using the monotonicity of $f$ and the inequality $\rho<1 / 2 n$ (cf. Proposition 3.1), we obtain

$$
\begin{aligned}
\left|\frac{f^{(\ell)}(\rho)}{\ell !}\right| & =\left(\rho+\frac{1}{n^{3 / 2}}\right)^{k+\ell} n^{3 \ell / 2} \prod_{\nu=1}^{k} \frac{1}{1-\nu\left(\rho+\frac{1}{n^{3 / 2}}\right)} \\
& \leq\left(\frac{1}{2 n}\right)^{k+\ell}\left(1+\frac{2}{\sqrt{n}}\right)^{k+\ell} n^{3 \ell / 2} \prod_{\nu=1}^{k} \frac{1}{1-\frac{\nu}{2 n}\left(1+\frac{2}{\sqrt{n}}\right)} .
\end{aligned}
$$

Thanks to (6), we get

$$
\begin{aligned}
\lim _{z \rightarrow \rho} \frac{M^{\prime}(z)}{A^{\prime}(z)} & \leq \rho^{r+2}\left(\begin{array}{c}
n-p \\
k+r
\end{array}\right)\left(\begin{array}{c}
k+r \\
k
\end{array}\right) 2^{k+r} k ! r ! \frac{f^{(\ell)}(\rho)}{(\ell-1) !} \lim _{z \rightarrow \rho} \frac{K^{\prime}(z)}{A^{\prime}(z)} \\
& \leq \ell\left(\frac{1}{2 n}\right)^{k+\ell+r+2} 2^{k+r}(n)_{k} n^{r}\left(1+\frac{2}{\sqrt{n}}\right)^{k+\ell} n^{3 \ell / 2} \prod_{\nu=1}^{k} \frac{1}{1-\frac{\nu}{2 n}\left(1+\frac{2}{\sqrt{n}}\right)} \lim _{z \rightarrow \rho} \frac{K^{\prime}(z)}{A^{\prime}(z)} \\
& \leq \ell e^{2} 2^{-\ell-2} \cdot n^{\ell / 2-2}\left(1+\frac{2}{\sqrt{n}}\right)^{k} \prod_{\nu=1}^{k} \frac{1-\frac{\nu}{n}}{1-\frac{\nu}{2 n}\left(1+\frac{2}{\sqrt{n}}\right)} \lim _{z \rightarrow \rho} \frac{K^{\prime}(z)}{A^{\prime}(z)}
\end{aligned}
$$

The last product is bounded by 1 and if $k=\mathcal{O}(\sqrt{n})$, then $\left(1+\frac{2}{\sqrt{n}}\right)^{k}$ is bounded as well. In this case we get

$$
\lim _{z \rightarrow \rho} \frac{M^{\prime}(z)}{A^{\prime}(z)}=\mathcal{O}\left(\ell 2^{-\ell} n^{\ell / 2-2} \lim _{z \rightarrow \rho} \frac{K^{\prime}(z)}{A^{\prime}(z)}\right)
$$

If $k>4 \sqrt{n}$ then it suffices to show that $\left(1+\frac{2}{\sqrt{n}}\right)^{k} n^{3 \ell / 2} \prod_{\nu=1}^{k} \frac{1}{1-\frac{\nu}{2 n}\left(1+\frac{2}{\sqrt{n}}\right)}$ is bounded. We proceed as follows: First observe that

$$
\frac{1-\frac{\nu}{n}}{1-\frac{\nu}{2 n}\left(1+\frac{2}{\sqrt{n}}\right)}<1-\frac{\nu}{2 n}\left(1-\frac{2}{\sqrt{n}}\right)
$$

and thus we can write

$$
\begin{aligned}
\left(1+\frac{2}{\sqrt{n}}\right)^{k} \prod_{\nu=1}^{k} \frac{1-\frac{\nu}{n}}{1-\frac{\nu}{2 n}\left(1+\frac{2}{\sqrt{n}}\right)}= & \left(1+\frac{2}{\sqrt{n}}\right)^{4\lfloor\sqrt{n}\rfloor} \prod_{\nu=1}^{4\lfloor\sqrt{n}\rfloor} \frac{1-\frac{\nu}{n}}{1-\frac{\nu}{2 n}\left(1+\frac{2}{\sqrt{n}}\right)} \\
& \times\left(1+\frac{2}{\sqrt{n}}\right)^{k-4\lfloor\sqrt{n}\rfloor} \prod_{\nu=4\lfloor\sqrt{n}\rfloor+1}^{k} \frac{1-\frac{\nu}{n}}{1-\frac{\nu}{2 n}\left(1+\frac{2}{\sqrt{n}}\right)} \\
\leq & e^{8}\left[\left(1+\frac{2}{\sqrt{n}}\right)\left(1-\frac{2}{\sqrt{n}}\left(1-\frac{2}{\sqrt{n}}\right)\right)\right]^{k-4\lfloor\sqrt{n}\rfloor} \\
& =e^{8}\left(1+\frac{8}{n^{3 / 2}}\right)^{k-4\lfloor\sqrt{n}\rfloor}=\mathcal{O}(1) .
\end{aligned}
$$

Finally, if we let $n$ tend to infinity and apply Lemma 5.3 , we get

$$
\mu_{n}\left(\mathcal{M}_{k, \ell, r}^{\Gamma}\right)=\mathcal{O}\left(r \ell^{2} 2^{r-\frac{3 \ell-1}{2}} n^{-3 / 2}\right)=\mathcal{O}\left(\frac{1}{n^{3 / 2}}\right) .
$$


Lemma 5.5. The limiting ratio of trees with fewer than $n^{1 / 4}$ different labels appearing on the first level leaves is $\mathcal{O}\left(\frac{1}{\sqrt{n}}\right)$.

Proof. The generating function of trees with exactly $k$ different labels appearing on the first level (with no occurrence of a variable and its negation) is given by

$$
G_{k}(z)=\left(\begin{array}{l}
n \\
k
\end{array}\right) 2^{k} k ! z \prod_{m=0}^{k} \frac{z}{1-m z-\hat{B}(z)},
$$

and therefore, their limiting ratio is given by

$$
\begin{aligned}
\lim _{z \rightarrow \rho} \frac{G_{k}^{\prime}(z)}{A^{\prime}(z)} & =\left(\begin{array}{l}
n \\
k
\end{array}\right) 2^{k} k ! \rho \prod_{m=0}^{k} \frac{\rho}{1-m \rho-\hat{B}(\rho)} \sum_{m=0}^{k} \frac{1}{1-m \rho-\hat{B}(\rho)} \cdot \lim _{z \rightarrow \rho} \frac{\hat{B}^{\prime}(z)}{A^{\prime}(z)} \\
& \leq \frac{1}{4 n} \prod_{m=0}^{k-1}\left(1-\frac{m}{n}\right) \prod_{m=0}^{k} \frac{1}{1-\frac{m}{2 n}-\frac{1}{\sqrt{2 n}}}\left(\sum_{m=0}^{k} \frac{1}{1-\frac{m}{2 n}-\frac{1}{\sqrt{2 n}}}\right) \\
& \leq \frac{1}{4 n}\left(\frac{1}{1-\frac{k}{2 n}-\frac{1}{\sqrt{2 n}}}\right)^{k+1}(k+1) \frac{1}{1-\frac{k}{2 n}-\frac{1}{\sqrt{2 n}}}=\mathcal{O}\left(\frac{k}{n}\right) .
\end{aligned}
$$

Summing up over all $k$ from 1 to $\left\lfloor n^{1 / 4}\right\rfloor$ yields the result.

Lemma 5.6. The limiting ratio of the family of all trees where the root has more than $n^{1 / 8}$ non-leaf subtrees is $\Theta\left(\frac{n^{1 / 8}}{2^{n^{1 / 8}}}\right)$.

Proof. The generating function of the trees with exactly $\ell$ non-leaf subtrees is

$$
H_{\ell}(z)=2 z \frac{\hat{B}^{\ell}(z)}{(1-2 n z)^{\ell+1}} .
$$

Therefore, the limiting ratio of trees with exactly $\ell$ non-leaf subtrees is given by

$$
\begin{aligned}
\lim _{z \rightarrow \rho} \frac{H_{\ell}^{\prime}(z)}{A^{\prime}(z)} & =\frac{1}{n} \frac{\ell \hat{B}^{\ell-1}(\rho)}{(1-2 n \rho)^{\ell+1}} \cdot \lim _{z \rightarrow \rho} \frac{\hat{B}^{\prime}(z)}{A^{\prime}(z)} \sim \frac{1}{2 n} \frac{\ell\left(\frac{1}{\sqrt{2 n}}\right)^{\ell-1}}{\left(\sqrt{\frac{2}{n}}\right)^{\ell+1}} \\
& \sim \frac{1}{2 n} \frac{\ell\left(\frac{1}{\sqrt{2 n}}\right)^{\ell-1}}{\left(\sqrt{\frac{2}{n}}\right)^{\ell+1}} \sim \frac{\ell}{2^{\ell+1}}, \text { as } n \rightarrow \infty .
\end{aligned}
$$

This implies that the limiting ratio of trees with more than $n^{1 / 8}$ non-leaf subtrees is given by

$$
\sum_{\ell \geq n^{1 / 8}} \frac{\ell}{2^{\ell+1}}=\Theta\left(\frac{n^{1 / 8}}{2^{n^{1 / 8}}}\right) .
$$

\section{TAutologies}

This section deals with tautologic trees and states the first statement of Theorem 2.4 about the probability of constant functions. This is not only a particular case, but also the first step to prove the whole theorem. The first subsection is devoted to prove the lower bound, the second one to prove the upper bound and the last one to prove that almost all tautologies have a very simple shape.

6.1. A non-negligible family of tautologies. In this section, we define a set of "simple" tautologies and find a lower bound for the limiting ratio of this family of tautologies.

Definition 6.1. A simple tautology (resp. a simple contradiction) realized by the variable $x$ is an $\vee$-rooted (resp. $\wedge$-rooted) tree such that both of the labels $x$ and $\bar{x}$ appear on the first level of the tree. The set of simple tautologies will be denoted by $\mathcal{S}_{n}$, its complement by $\overline{\mathcal{S}_{n}}$. 
Lemma 6.2. There exists a constant $\alpha$ such that, for all $n \geq 0$,

$$
0<\alpha \leq \mathbb{P}_{n} \text { (True). }
$$

Proof. Let $\mathcal{R}$ be the family of trees such that their root is labelled by $\vee$, containing at least $\lfloor\sqrt{n}\rfloor$ leaves on the first level and a single $\wedge$-rooted tree attached to the root. Furthermore, the $\lfloor\sqrt{n}\rfloor$ leftmost children of the root are leaves. The generating function $R(z)$ satisfies

$$
R(z)=\frac{z \cdot(2 n z)^{\lfloor\sqrt{n}\rfloor}}{1-2 n z} \cdot \hat{B}(z) \cdot \frac{1}{1-2 n z} .
$$

The generating function $R(z)$ has the same dominant singularity as $\hat{A}(z)$ and thus the same as $A(z)$. By using Lemma 4.2 we get

$$
\frac{R^{\prime}(z)}{A^{\prime}(z)}=\frac{\rho(2 n \rho)^{\lfloor\sqrt{n}\rfloor}}{(1-2 n \rho)^{2}} \cdot \lim _{z \rightarrow \rho} \frac{\hat{B}^{\prime}(z)}{A^{\prime}(z)}=\frac{e^{-\sqrt{2}}}{8}+o(1) .
$$

Thus, the family $\mathcal{R}$ contains a positive ratio of all trees. In order to conclude, the last idea consists of using the birthday paradox (see e.g. [6] and the references therein) in order to prove that a positive ratio of trees from $\mathcal{R}$ is obtained by simple tautologies. The probability that among the $\lfloor\sqrt{n}\rfloor$ leftmost leaves, of the first level, there is no repetition of variables is $n(n-1) \cdot(n-\lfloor\sqrt{n}\rfloor+1) / n\lfloor\sqrt{n}\rfloor$. Thus by using Stirling formula this probability tends to $1 / e$. Consequently, for $n$ sufficiently large, with probability larger than $1 / 2$ there is at least one repetition of variables among the $\lfloor\sqrt{n}\rfloor$ leftmost leaves of the first level. Let us take into account the two first (from left to right) occurrences of the same variable, with probability $1 / 2$ they are labelled by two opposite literals, and thus the whole tree is a simple tautology.

Finally, when $n$ tends to infinity, the probability to be a simple tautology is positive. Furthermore, the previous results also hold for small value of $n$, which concludes the proof.

The following investigations aim at deriving a numerical lower bound for the probability of the set of simple tautologies. In order to get such a result, we utilized a computer algebra software to perform some routine calculations. In these instances, we will omit the details.

Let $\mathcal{E}^{k}$ be the set of trees rooted by $\vee$, with exactly $k$ leaves at depth 1 and at most 5 non-leaf subtrees. Let us take $k$ in $M:=\{\lfloor\sqrt{n}\rfloor, \ldots, 15\lfloor\sqrt{n}\rfloor\}$. For two generating functions $f, g \in \mathbb{R}[[z]]$, we write $f \prec g$ (resp. $f \succ g$ ) if $\left[z^{r}\right] f \leqslant\left[z^{r}\right] g$ (resp. $\left[z^{r}\right] f \geqslant\left[z^{r}\right] g$ ) for all $r \in \mathbb{N}$. The generating function of $\mathcal{E}=\bigcup_{k \in M} \mathcal{E}^{k}$ satisfies

$$
E(z) \succ \sum_{j=1}^{5} \sum_{k=\lfloor\sqrt{n}\rfloor}^{15\lfloor\sqrt{n}\rfloor} z \cdot(2 n z)^{k} \cdot \frac{(k+1)^{j} \hat{B}^{j}(z)}{j !} .
$$

The latter generating function is not exactly $E(z)$ because of the approximation $(k+1) \cdots(k+j) \sim(k+1)^{j}$. Using the approximated form to compute the limiting ration, we obtain after some computations: $\mu_{n}(\mathcal{E}) \geqslant 0.36618+o(1)$ for $n$ tending to infinity.

Let us now introduce two subfamilies of $\mathcal{E}^{k}$. The first family $\mathcal{E}_{1}^{k}$ contains all trees of $\mathcal{E}^{k}$ such that

(a) the set $\mathcal{L}$ of all labels appearing among the $\lfloor\sqrt{n}\rfloor$ leftmost leaves at depth 1 is of cardinality at least $\sqrt{n} / 2$,

(b) and the $k-\lfloor\sqrt{n}\rfloor$ rightmost leaves of depth 1 are labelled by literals whose negation does not belong to $\mathcal{L}$. The family $\mathcal{E}_{2}^{k}$ contains all trees of $\mathcal{E}^{k}$ such that

(a') the set $\mathcal{L}^{\prime}$ of all labels appearing among the $\lfloor\sqrt{n}\rfloor$ leftmost leaves at depth 1 is of cardinality at most $\sqrt{n} / 2$

(b) and the $k-\lfloor\sqrt{n}\rfloor$ rightmost leaves of depth 1 are labelled by literals whose negation does not belong to $\mathcal{L}^{\prime}$.

The following lemma comes from the fact that a tautology which is not simple fulfills either condition (a) or (a') and always satisfies condition (b) since a variable and its negation cannot appear in the first level of the tree. Therefore, a non-simple tautology is either in $\mathcal{E}_{1}^{k}$ or in $\mathcal{E}_{2}^{k}$. The same applies to trees which do not even represent tautologies. Thus we obtain the following result:

Lemma 6.3. We have $\mathcal{E} \cap \overline{\mathcal{S}_{n}} \subseteq \bigcup_{k \in M}\left(\mathcal{E}_{1}^{k} \cup \mathcal{E}_{2}^{k}\right)$.

Let us now estimate the sizes of the sets $\mathcal{E}_{1}^{k}$ and $\mathcal{E}_{2}^{k}$. We get an upper bound by multiple counting: Let $E_{1}^{k}(z)$ (resp. $E_{2}^{k}(z)$ ) be the generating functions of $\mathcal{E}_{1}^{k}$ (resp. $\mathcal{E}_{2}^{k}$ ). Then

$$
E_{1}^{k}(z) \prec \sum_{j=1}^{5} z \cdot(2 n z)^{\sqrt{n}} \cdot\left(\left(2 n-\frac{\sqrt{n}}{2}\right) z\right)^{k-\sqrt{n}} \cdot \frac{(k+5)^{j} \hat{B}^{j}(z)}{j !} .
$$


Some multiple counting is done for the leftmost $\lfloor\sqrt{n}\rfloor$ leaves at depth 1 , because we did not restrict to labellings such that $|\mathcal{L}| \geq\lceil\sqrt{n} / 2\rceil$. Further over-counting appears by forbidding only $\lceil\sqrt{n} / 2\rceil$ labels instead of $|\mathcal{L}|$ for the rightmost $k-\lfloor\sqrt{n}\rfloor$ leaves. Using the function on the right-hand side of (11) for the computation of the limiting ratio, we get 0.24457 as an asymptotic upper bound for the limiting ratio of the family $\bigcup_{k \in M} \mathcal{E}_{1}^{k}$.

Furthermore,

$$
E_{2}^{k}(z) \prec \sum_{j=1}^{5}\left(\begin{array}{c}
n \\
\lfloor\sqrt{n} / 2\rfloor
\end{array}\right) 2^{\sqrt{n} / 2} \cdot z \cdot\left(\frac{\sqrt{n}}{2} z\right)^{\sqrt{n}} \cdot(2 n z)^{k-\sqrt{n}} \cdot \frac{(k+5)^{j} \hat{B}^{j}(z)}{j !} .
$$

Again we do some multiple counting in the same fashion as before. The function on the right-hand side of 12 permits us to conclude that the limiting ratio of the family $\bigcup_{k \in M} \mathcal{E}_{2}^{k}$ is of order $o(1)$, as $n \rightarrow \infty$.

Proposition 6.4. The limiting ratio of the set of simple tautologies is greater than $\alpha+o(1)$ where $\alpha:=0.12161$.

Proof. The previous lemma is equivalent to $\mathcal{E} \cap \mathcal{S} \supseteq \mathcal{E} \backslash \bigcup_{k \in M}\left(\mathcal{E}_{1}^{k} \cup \mathcal{E}_{2}^{k}\right)$. Thus, for $n \rightarrow \infty$ we have

$$
\mu_{n}\left(\mathcal{S}_{n}\right) \geq \mu_{n}\left(\mathcal{S}_{n} \cap \mathcal{E}\right) \geq \mu_{n}(\mathcal{E})-\left(\sum_{k=\sqrt{n}}^{15 \sqrt{n}} \mu_{n}\left(\mathcal{E}_{1}^{k}\right)+\mu_{n}\left(\mathcal{E}_{2}^{k}\right)\right) \geq 0.36618-0.24457+o(1)
$$

6.2. A non-negligible family of non-tautologies. In this section, we derive an upper bound for the probability of the set of tautologies.

Definition 6.5. Let $\breve{\mathcal{G}}_{k}$ be the family of $\vee$-rooted trees with exactly $k$ leaves on the first level, labelled by $k$ different literals $\alpha_{1}, \ldots, \alpha_{k}$ such that each variable can only appear positive or negative and whose non-leaf subtrees are all contradictions.

Note that an $\vee$-rooted tree in $\breve{\mathcal{G}}$ computes the function $\alpha_{1} \vee \ldots \vee \alpha_{k}$, and is therefore neither a tautology nor a contradiction.

Lemma 6.6. There exists a constant $\beta$ such that, for all $n \geq 0$,

$$
\mathbb{P}_{n}(\text { True }) \leq \beta<\frac{1}{2}
$$

Proof. The generating function of the tree family defined in Definition 6.5 is given by

$$
\check{G}_{k}(z)=\left(\begin{array}{l}
n \\
k
\end{array}\right) 2^{k} k ! z^{k+1} \frac{1}{(1-T(z))^{k+1}},
$$

and its limiting ratio is given by

$$
\lim _{z \rightarrow \rho} \frac{\check{G}^{\prime}(z)}{A^{\prime}(z)} \sim\left(\begin{array}{l}
n \\
k
\end{array}\right) 2^{k} k ! \rho^{k+1} \frac{k+1}{(1-T(\rho))^{k+1}} \lim _{z \rightarrow \rho} \frac{T^{\prime}(z)}{A^{\prime}(z)} .
$$

But Proposition 6.4 tells us that $\lim _{z \rightarrow \rho} \frac{T^{\prime}(z)}{A^{\prime}(z)} \geq \frac{\alpha}{2}$. Moreover, we know that $T(\rho)>0$ and therefore $\frac{1}{(1-T(\rho))^{k+1}}>1$. Therefore,

$$
\begin{aligned}
\lim _{z \rightarrow \rho} \frac{G^{\prime}(z)}{A^{\prime}(z)} & \geq\left(\begin{array}{l}
n \\
k
\end{array}\right) 2^{k} k !(k+1)\left(\frac{1}{2 n}\right)^{k+1} \frac{\alpha}{2} \\
& =\alpha \cdot \frac{n(n-1) \ldots(n-k+1)}{n^{k}} \frac{k+1}{4 n} \\
& \geq \alpha \cdot\left(1-\frac{k-1}{n}\right)^{k-1} \frac{k+1}{4 n} .
\end{aligned}
$$

If $k=\Theta(\sqrt{n})$, then the right-hand side of the above inequality is of order $\Theta(1 / \sqrt{n})$, and the limiting ratio of $\check{\mathcal{G}}_{k}$ is thus larger than $c_{k} / \sqrt{n}$ where $c_{k}$ is a positive constant. Thus the limiting ratio of the family $\bigcup_{k=\lfloor\sqrt{n}\rfloor}^{2\lfloor\sqrt{n}\rfloor} \check{\mathcal{G}}_{k}$ is bounded from below by a positive constant $c$. We therefore have proved Lemma 6.6, and $\beta=1 / 2-c$. 
6.3. Almost every tautology is simple. In general, it is not easy to describe precisely the shape of a tautologic tree, but according to our distribution on trees, almost every tautology is "simple".

Theorem 6.7. Asymptotically almost every tautology is a simple tautology, i.e.

$$
\mathbb{P}_{n}(\text { True })=\mu_{n}\left(\mathcal{S}_{n}\right)+o(1) \text {, as } n \rightarrow \infty .
$$

Proof. To prove Theorem 6.7, we will consider the family of non-simple tautologies, study the structure of its elements and show that its limiting ratio tends to zero when $n$ tends to infinity.

Let us consider $\mathcal{N}_{n}=\mathcal{T}_{n} \backslash \mathcal{S}_{n}$ the set of tautologies which are not simple. Let $t \in \mathcal{N}_{n}$, with $\ell$ non-leaf subtrees $A_{1}, \ldots, A_{\ell}$ and $k$ different labels $\alpha_{1}, \ldots, \alpha_{k}$ appearing in the first level. Since $t$ is not a simple tautology, the set $L=\left\{\alpha_{1}, \ldots, \alpha_{k}\right\}$ cannot contain both a variable and its negation. For each $i \in\{1, \ldots, \ell\}$, the tree $A_{i}$ has some of its leaves in its first level labelled by labels in $L$ and others carrying new labels. What will be called a "new variable" in the following is the label of a leaf in the first level of one of the $A_{i}$ if it does not belong to $L$.

Let us show that there exists at least one $i \in\{1, \ldots, \ell\}$ such that $A_{i}$ has at most $\ell-1$ new variables.

Let us assume that for all $i \in\{1, \ldots, \ell\}, A_{i}$ has at least $\ell$ new variables in the first level. We assign one of those new variables, say $\nu_{1}$ and belonging to $A_{1}$, to False. Then $A_{1}$ computes False. But, there must exist $A_{(2)} \in\left\{A_{2}, \ldots, A_{\ell}\right\}$ which is not a contradiction for this assignment, because if $A_{1}=A_{2}=\ldots=A_{\ell} \equiv$ False for $\nu_{1}=\mathrm{False}$, then the whole tree $t$ would compute $\alpha_{1} \vee \ldots \vee \alpha_{k}$ and would thus not be a tautology.

Let us iterate this algorithm: After step $s-1 \leq \ell$, we have assigned $\nu_{1}=\nu_{2}=\ldots=\nu_{s-1}=$ False and at least $s-1$ trees of $\left\{A_{1}, \ldots A_{\ell}\right\}$ compute False. At step $s$ note that at least one of the remaining subtrees must still not be a contradiction: Let us call this tree $A_{(s)}$. It has at least $\ell$ new variables and we have assigned $s-1 \leq \ell$ variables to $\mathrm{False}$ so far. Therefore we still have free new variables among the new variables of $A_{(s)}$, and we can assign one, denoted by $\nu_{s}$, to False.

After the $\ell^{\text {th }}$ step, we have found an assignment of variables different from those in $L$ such that all trees of $\left\{A_{1}, \ldots, A_{\ell}\right\}$ compute False. Thus $t$ computes $\alpha_{1} \vee \ldots \vee \alpha_{k}$, which is impossible since $t$ is a tautology.

Therefore, there exist at least one $i \in\{1, \ldots, \ell\}$ such that $A_{i}$ has at most $\ell-1$ new variables in the first level.

It means that $\mathcal{N}_{n} \subseteq \bigcup_{k=0}^{n} \bigcup_{\ell=0}^{\infty} \bigcup_{r=0}^{\ell-1} \mathcal{M}_{k, \ell, r}^{\emptyset}$ (cf. Section 5). Let us decompose this union into three distinct unions:

$$
\begin{aligned}
& \bigcup_{k=0}^{n} \bigcup_{\ell=0}^{\infty} \bigcup_{r=0}^{\ell-1} \mathcal{M}_{k, \ell, r}^{\emptyset} \\
& \quad=\left(\bigcup_{k=0}^{\left\lfloor n^{1 / 4}\right\rfloor} \bigcup_{\ell=0}^{\infty} \bigcup_{r=0}^{\ell-1} \mathcal{M}_{k, \ell, r}^{\emptyset}\right) \cup\left(\bigcup_{k=\left\lfloor n^{1 / 4}\right\rfloor}^{n} \bigcup_{\ell=\left\lfloor n^{1 / 8}\right\rfloor}^{\infty} \bigcup_{r=0}^{\ell-1} \mathcal{M}_{k, \ell, r}^{\emptyset}\right) \cup\left(\bigcup_{k=\left\lfloor n^{1 / 4}\right\rfloor}^{n} \bigcup_{\ell=0}^{\left\lfloor n^{1 / 8}\right\rfloor} \bigcup_{r=0}^{\ell-1} \mathcal{M}_{k, \ell, r}^{\emptyset}\right) .
\end{aligned}
$$

Thanks to Lemma 5.5 the first term has a limiting ratio tending to zero as $n$ tends to infinity; Lemma 5.6 guarantees that the second term has also a limiting ratio tending to zero, and by Lemma 5.4 , the third term satisfies

$$
\mu_{n}\left(\left(\bigcup_{k=\left\lfloor n^{1 / 4}\right\rfloor}^{n} \bigcup_{\ell=0}^{\left\lfloor n^{1 / 8}\right\rfloor} \bigcup_{r=0}^{\ell-1} \mathcal{M}_{k, \ell, r}^{\emptyset}\right)\right)=\mathcal{O}\left(\left(n-n^{1 / 4}\right)\left(n^{1 / 8}\right)^{2} \frac{1}{n^{3 / 2}}\right)=\mathcal{O}\left(\frac{1}{n^{1 / 4}}\right)
$$

and is thus also tending to zero when $n$ tends to infinity. Thus, $\mu_{n}\left(\mathcal{N}_{n} \backslash \mathcal{S}_{n}\right)=o(1)$ and Theorem 6.7 is proved.

\section{Probability of functions larger than a fixed $f_{0}$}

In this section we investigate the probability of all the functions that are "larger" than a fixed given Boolean function. The result will enable us to prove the second statement of Theorem 2.4 about literal functions, proved in Section 8 .

Definition 7.1. Let $f$ and $g$ be two Boolean functions of $n$ variables, we say that $g \geq f$ if and only if, $g\left(x_{1}, \ldots, x_{n}\right) \geq$ $f\left(x_{1}, \ldots, x_{n}\right)$ for all $\left(x_{1}, \ldots, x_{n}\right) \in\{0,1\}^{n}$.

In the following we fix a non-constant Boolean function $f_{0}$ and estimate $\mathbb{P}_{n}\left(f \geq f_{0}\right)$. We first consider the case $f_{0}=x_{1} \wedge \ldots \wedge x_{p}$ and then generalize the result to any Boolean function. 
Proposition 7.2. Choose $n_{0}$ and a non-constant function $f_{0} \in \mathcal{F}_{n_{0}}$. Moreover, denote the natural extension of $f_{0}$ to $\mathcal{F}_{n}$ for $n>n_{0}$, i.e. the function $\left(x_{1}, \ldots, x_{n_{0}}, x_{n_{0}+1}, \ldots, x_{n}\right) \mapsto f_{0}\left(x_{1}, \ldots, x_{n_{0}}\right)$, by $f_{0}$ as well. Denote by $F$ a random Boolean function having law $\mathbb{P}_{n}$, i.e. $\mathbb{P}(F=f)=\mathbb{P}_{n}(f)$ for all Boolean function $f$. Then

$$
\mathbb{P}\left(F \geq f_{0}\right)=\mathbb{P}_{n} \text { (True) }(1+o(1)) \text {, as } n \text { tends to infinity. }
$$

The remaining part of this section is devoted to the proof of Proposition 7.2 We prove this proposition in two steps: First, assume that $f_{0}$ is a conjunction of literals, and then we extend the proof to obtain the general result.

7.1. Case I: $\boldsymbol{f}_{\mathbf{0}}$ is a conjunction of literals. Let $f_{0}=\gamma_{1} \wedge \ldots \wedge \gamma_{p}$, where the $\gamma_{i}$ 's are literals. First, let us remark that $\mathbb{P}\left(F \geq f_{0}\right) \geq \mathbb{P}_{n}$ (True) $\geq \alpha>0$. Let us consider an associative tree $t$ computing a Boolean function which is larger than $f_{0}$ and not a tautology.

The family of trees with no leaf on the first level has a limiting ratio which is asymptotically equal to $1 / n \sqrt{2 n}$ when $n$ tends to infinity ( $c f$. Proposition 4.3). It is thus negligible compared with $\mathbb{P}$ (True). Thus, we can assume that $t$ has at least one leaf on the first level.

Consider first the case where $t$ is rooted by an $\vee$ :

- Let us first assume that there exists one leaf on the first level of $t$, labelled by one of the $\gamma_{i}$. The family of trees with at least one leaf on the first level and with a label from the set $\Gamma=\left\{\gamma_{1}, \ldots, \gamma_{p}\right\}$ has a limiting ratio equivalent to $p \sqrt{2 / n}$, in view of Proposition 4.4 The limiting ratio of such trees is thus negligible compared with the limiting ratio of the set of tautologies. We can thus neglect this family.

- Let us assume that $t$ has no leaf on the first level labelled by a literal chosen in $\Gamma=\left\{\gamma_{1}, \ldots, \gamma_{p}\right\}$. Let us denote by $k$ the number of different labels, denoted by $\alpha_{1}, \ldots, \alpha_{k}$, appearing on the first level of $t$ and by $\ell$ the number of its non-leaf subtrees, denoted by $A_{1}, \ldots, A_{\ell}$. Observe that since $t$ is not a tautology, the labels appearing on the first level of $t$ cannot contain a variable and its negation. The subtrees $A_{1}, \ldots, A_{\ell}$ have themselves leaves on their first level (i.e. on the second level of $t$ ), and those leaves are labelled either by "old variables", i.e. by literal chosen from $\mathcal{O}=\left\{\alpha_{1}, \ldots, \alpha_{k}, \gamma_{1}, \ldots, \gamma_{p}\right\}$ and their negations, or by "new variables", i.e by other literals. Assume that for all $i \in\{1, \ldots, \ell\}, A_{i}$ has at least $\ell$ different new variables appearing on its first level. Thus, since each $A_{i}$ is rooted by an $\wedge$, we can find an assignment of the variables $\left\{x_{1}, \ldots, x_{n}\right\} \backslash \mathcal{O}$ such that all $A_{i}$ compute False for this assignment. Assign then all the leaves on the first level of $t$ to False and $\gamma_{1}, \ldots, \gamma_{p}$ to True. Then $t$ computes False while $\gamma_{1} \wedge \ldots \wedge \gamma_{p}$ takes the value True for this assignment. But this is impossible!

Thus, there exists at least one $A_{i}$ which has fewer than $\ell$ new variables on its first level. This means that $t$ belongs to the set $\bigcup_{r=0}^{\ell-1} \mathcal{M}_{k, \ell, r}^{\Gamma}$.

The limiting ratio of such trees is thus less than the limiting ratio of $\bigcup_{k, \ell \geq 0} \bigcup_{r=0}^{\ell-1} \mathcal{M}_{k, \ell, r}^{\Gamma}$, and thanks to the results proved Section 5 we have

$$
\mu_{n}\left(\bigcup_{k, \ell \geq 0} \bigcup_{r=0}^{\ell-1} \mathcal{M}_{k, \ell, r}^{\Gamma}\right)=\mathcal{O}\left(\left(n-n^{1 / 4}\right)\left(n^{1 / 8}\right)^{2} \frac{1}{n^{3 / 2}}\right)=\mathcal{O}\left(\frac{1}{n^{1 / 4}}\right)
$$

and this family is also negligible in in front of tautologies.

If $t$ is rooted by an $\wedge$, then its first level leaves have labels chosen from the set $\Gamma=\left\{\gamma_{1}, \ldots, \gamma_{p}\right\}$. The family of trees with first level leaves labelled in $\left\{\gamma_{1}, \ldots, \gamma_{p}\right\}$ has generating function

$$
H_{\Gamma}(z)=2 n z+2 z \frac{(\hat{B}(z)+p z)^{2}}{1-\hat{B}(z)-p z}
$$

and its limiting ratio is asymptotically equal to $1 / n \sqrt{2 n}$. It is thus negligible compared with the set of tautologies. We have thus proved that

as $n$ tends to infinity.

$$
\mathbb{P}\left(F \geq \gamma_{1} \wedge \ldots \wedge \gamma_{p}\right) \sim \mathbb{P}_{n}(\text { True })
$$

7.2. Case II: $f_{0}$ is any non-constant Boolean function. All Boolean functions $f_{0}$ can be written as $f_{0}=$ $\left(\gamma_{1} \wedge \ldots \wedge \gamma_{p}\right) \vee g_{0}$ for some integer $p \geq 1$, some literals $\left\{\gamma_{1}, \ldots, \gamma_{p}\right\}$, and some Boolean function $g_{0}$. Thus,

$$
\mathbb{P}_{n}(\text { True }) \leq \mathbb{P}\left(F \geq f_{0}\right) \leq \mathbb{P}\left(F \geq \gamma_{1} \wedge \ldots \wedge \gamma_{p}\right) \sim \mathbb{P}_{n}(\text { True })
$$

and Proposition 7.2 is proved. 


\section{Literals}

The aim of this section is to estimate the probability of a literal Boolean function i.e. a function of the shape $\left(\left(x_{1}, \ldots, x_{n}\right) \mapsto x\right)$ where $x$ is a literal among $\left\{x_{1}, \bar{x}_{1}, \ldots, x_{n}, \bar{x}_{n}\right\}$, therefore proving the second statement of Theorem 2.4 As for tautologies, we will prove that a typical tree computing this function has a very simple shape.

Definition 8.1. A tree $t$ is a simple $x$ tree if it is rooted by an $\vee$ (resp. $\wedge$ ), with one single leaf on the first level, labelled by $x$ and with one non-leaf subtree which is a tautology (resp. a contradiction). We denote by $\mathcal{X}$ the family of such trees and and by $X_{m}$ the number of simple $x$ trees of size $m$.

Lemma 8.2. For $n$ tending to infinity, the limiting ratio of the set simple $x$ trees satisfies

$$
\mu_{n}(\mathcal{X}) \sim \frac{\mathbb{P}_{n}(\text { True })}{n^{2}}
$$

Proof. The generating function associated with the set of simple $x$ trees is given by the following generating function:

$$
X(z)=4 z^{2} T(z)
$$

where the 4 factor contains the choice of the label of the root and the order of its first and second child. Therefore,

$$
\mu_{n}(\mathcal{X})=\lim _{z \rightarrow \rho} \frac{X^{\prime}(z)}{A^{\prime}(z)}=4 \rho^{2} \lim _{z \rightarrow \rho} \frac{T^{\prime}(z)}{A^{\prime}(z)} .
$$

Observing that $\frac{T^{\prime}(z)}{A^{\prime}(z)}$ tends to $\mathbb{P}_{n}$ (True) when $z$ tends to $\rho$ and that $\rho \sim 1 / 2 n$ when $n$ tends to infinity (cf. Proposition 3.1 permits to complete the proof.

Theorem 8.3. For $n$ tending to infinity,

$$
\mathbb{P}_{n}(x) \sim \mu_{n}(\mathcal{X})
$$

Proof. Thanks to Lemma 8.2 and Proposition 6.4 we know that

$$
\mathbb{P}_{n}(x) \geq \frac{\mathbb{P}_{n}(\text { True })}{2 n^{2}} \geq \frac{\alpha}{2 n^{2}}
$$

when $n$ tends to infinity. Let $t$ be a tree computing $x$. Let us assume that it is rooted by an $\wedge$ (the case of an $\checkmark$-rooted tree would be treated in the very same way). The family of trees computing $x$ with no leaf on the first level has the same limiting ratio for all $x \in\left\{x_{1}, \bar{x}_{1}, \ldots, x_{n}, \bar{x}_{n}\right\}$. Therefore, if we denote this family by $\mathcal{A}_{x}^{(0)}$, its limiting ratio satisfies ( $c f$. Proposition 4.3)

$$
2 n \mu_{n}\left(\mathcal{A}_{x}^{(0)}\right) \leq \mu_{n}\left(\mathcal{A}^{(0)}\right) \sim \frac{1}{n \sqrt{2 n}}
$$

Thus, the limiting ratio of trees with no leaf on the first level, computing $x$ has order $\mathcal{O}\left(n^{-5 / 2}\right)$. This family is negligible in comparison with the family of simple $x$ trees. Thus we can focus on the family $\mathcal{T}_{x}$ of trees having at least one leaf on the first level.

The leaves on the first level of $t$ have to be labelled by $x$ since $t$ computes $x$. And the non-leaf subtrees calculate functions that are larger than $x$ (in the sense of Defintion 7.1). Thus, a tree computing $x$ is almost surely an $\wedge$-rooted (resp. $\vee$-rooted) tree with leaves on the first level labelled by $x$ and with non-leaf subtrees larger than $x$ (resp. smaller than $x$ ).

Let us denote by $L_{x}(z)$ the generating function of trees larger than $x$. In view of Proposition 7.2 , we have

$$
\lim _{z \rightarrow \rho} \frac{L_{x}^{\prime}(z)}{A^{\prime}(z)} \sim \mathbb{P}_{n} \text { (True), when } n \rightarrow \infty .
$$

Note also that, by symmetry, the family of trees smaller than $x$ has the same generating function. Thus, the above described family of trees has the same limiting ratio as that of all trees computing $x$ and its generating function is given by

The limiting ratio is then

$$
T_{x}(z)=\frac{2 z^{2}}{\left(1-\left(L_{x}(z)-z\right)\right)\left(1-L_{x}(z)\right)}-2 z^{2}
$$

$$
\mu_{n}\left(\mathcal{T}_{x}\right) \sim 2 \rho^{2}\left(\frac{1}{\left(1-\left(L_{x}(\rho)-\rho\right)\right)^{2}\left(1-L_{x}(\rho)\right)}+\frac{1}{\left(1-\left(L_{x}(\rho)-\rho\right)\right)\left(1-L_{x}(\rho)\right)^{2}}\right) \lim _{z \rightarrow \rho} \frac{L_{x}^{\prime}(z)}{A^{\prime}(z)} \sim 4 \rho^{2} \mathbb{P}_{n}(\operatorname{True})
$$


as $n \rightarrow \infty$. Thus,

$$
\mathbb{P}_{n}(x) \sim \mu_{n}(\mathcal{X}) \sim \frac{\mathbb{P}_{n}(\text { True })}{n^{2}}, \text { as } n \rightarrow \infty .
$$

\section{General Case: minimal trees and expansions.}

In this last section we prove the last statement of Theorem 2.4. We use different expansions of trees, as it was done in other random Boolean tree models (cf. [11] for implication random trees and [15] for and/or trees). The first subsection defines the expansions, the second subsection states an asymptotic lower bound for $\mathbb{P}_{n}(f)$, and the third subsection states an asymptotic upper bound and thus completes the proof of Theorem 2.4

\subsection{Expansions.}

Definition 9.1. Let $t$ be an associative tree. The tree given by adding a new subtree te to an internal node $\nu$ of $t$ is called an expansion of $t$. An expansion is valid if the expanded tree computes the same function as $t$.

- The expansion is called a tautology expansion (resp. a contradiction expansion) if the added tree $t_{e}$ is a tautology (resp. a contradiction) and if $\nu$ is labelled by $a \wedge($ resp. $\vee)$. Obviously, such an expansion is valid.

- It is called a $\hat{\boldsymbol{B}}$-expansion if the added tree $t_{e}$ is not a single leaf.

Given a family of trees $\mathcal{T}$, we denote by $E(\mathcal{T})$ the set of trees obtained by a single tautology expansion of a tree in $\mathcal{T}$, by $E^{k}(\mathcal{T})$ the set of trees obtained by $k$ successive tautology expansions done at (not necessarily distinct) vertices of a tree in $\mathcal{T}$, and by $E^{\geq k}(\mathcal{T})$ the set of all trees obtained by at least $k$ successive tautology expansions done at (not necessarily distinct) vertices of a tree in $\mathcal{T}$. Finally, we set $E^{*}(\mathcal{T}):=\bigcup_{k \geq 1} E^{k}(\mathcal{T})$.

If the considered expansions are $\hat{B}$-expansions, we change the above notation by replacing $E$ by $E_{\hat{B}}$.

Remark: Whatever type of expansion (tautology or $\hat{B}$ ) we consider, note that nesting expansions (adding $t_{e}$ to $t$, then expanding $t_{e}$, and so on) does not generate new structures, since this can always be realized by a single expansion. Therefore, requiring that the expansions are done at the vertices of the original tree is no restriction.

Remark: For every family $\mathcal{T}$ of trees the inclusion $E^{*}(\mathcal{T}) \subseteq E_{\hat{B}}^{*}(\mathcal{T})$ holds.

\subsection{Tautology expansions.}

Proposition 9.2. For $n$ tending to infinity, the limiting ratio of $E^{*}\left(\mathcal{M}_{f}\right)$ is asymptotically equal to the limiting ratio of $E\left(\mathcal{M}_{f}\right)$. Furthermore,

$$
\mu_{n}\left(E\left(\mathcal{M}_{f}\right)\right)=\Theta\left(\frac{1}{n^{L(f)}}\right) \text {, as } n \rightarrow \infty .
$$

Since every tree in $E^{*}\left(\mathcal{M}_{f}\right)$ computes $f$, this implies

$$
\mathbb{P}_{n}(f) \geq \mu_{n}\left(E^{*}\left(\mathcal{M}_{f}\right)\right)=\Theta\left(\frac{1}{n^{L(f)}}\right) .
$$

Proof. Let $\Phi_{k}(z)$ be the generating function of $\left.E^{k}\left(\mathcal{M}_{f}\right)\right)$. Given a tree $t_{e}$, the number of places where $t_{e}$ can be added to a given minimal tree $t$ is

$$
P_{t}=\sum_{i \text { internal node of } t}(d(i)+1)
$$

where $d(i)$ is the number of children of the internal node $i$. Let $i_{t}$ denote the number of internal nodes of $t$ and $|t|$ the size of $t$. Then $P_{t}=i_{t}+|t|-1$. Since $t$ is minimal $(i . e .|t|=L(f))$ and since $1 \leq i_{t} \leq\left\lfloor\frac{L(f)}{2}\right\rfloor$, we have $L(f) \leq P_{t} \leq \frac{3 L(f)}{2}$ which yields

$$
m_{f} z^{L(f)} L(f) T(z) \prec \Phi_{1}(z) \prec m_{f} z^{L(f)} \frac{3 L(f)}{2} T(z),
$$

and thus

$$
m_{f} L(f) \rho^{L(f)} \lim _{z \rightarrow \rho} \frac{T^{\prime}(z)}{A^{\prime}(z)} \leq \mu_{n}\left(E\left(\mathcal{M}_{f}\right)\right) \leq m_{f} \frac{3 L(f)}{2} \rho^{L(f)} \lim _{z \rightarrow \rho} \frac{T^{\prime}(z)}{A^{\prime}(z)} .
$$


From Section 6 we know that $0<\alpha \leq \mathbb{P}_{n}(\operatorname{True})=\lim _{z \rightarrow \rho} \frac{T^{\prime}(z)}{A^{\prime}(z)} \leq \beta$ and since $\rho \sim \frac{1}{2 n}$ when $n$ tends to infinity, we get

$$
\mu_{n}\left(E\left(\mathcal{M}_{f}\right)\right)=\Theta\left(\frac{1}{n^{L(f)}}\right)
$$

If we do $k$ successive expansions in a minimal tree, we have at most $\lfloor 3 L(f) / 2\rfloor$ different places for the first one, $\lfloor 3 L(f) / 2\rfloor+1$ for the second one, and so on. We thus have the following inequality:

$$
\Phi_{k}(z) \prec m_{f} z^{L(f)}\left(\begin{array}{c}
\lfloor 3 L(f) / 2\rfloor+k-1 \\
k
\end{array}\right) T(z)^{k}
$$

and thus

$$
\begin{aligned}
\mu_{n}\left(E^{k}\left(\mathcal{M}_{f}\right)\right)=\lim _{z \rightarrow \rho} \frac{\Phi_{k}^{\prime}(z)}{A^{\prime}(z)} & \leq m_{f} \rho^{L(f)}\left(\begin{array}{c}
\lfloor 3 L(f) / 2\rfloor+k-1 \\
k
\end{array}\right) k T(\rho)^{k-1} \lim _{z \rightarrow \rho} \frac{T^{\prime}(z)}{A^{\prime}(z)} \\
& \leq \beta m_{f} \rho^{L(f)}\left(\begin{array}{c}
\lfloor 3 L(f) / 2\rfloor+k-1 \\
k
\end{array}\right) k T(\rho)^{k-1}
\end{aligned}
$$

for all $n \geq 0$. Hence

$$
\mu_{n}\left(E^{k \geq 2}\left(\mathcal{M}_{f}\right)\right) \leq \beta m_{f} \rho^{L(f)}\lfloor 3 L(f) / 2\rfloor\left(\frac{1}{\left.(1-T(\rho))^{\left\lfloor^{3 L(f) / 2\rfloor+1}\right.}-1\right)}\right.
$$

where we used $\sum_{k \geq 2}\left(\begin{array}{c}C+k-1 \\ k\end{array}\right) k z^{k-1}=\frac{C}{(1-z)^{C+1}}-C$ which is an immediate consequence of

$$
\sum_{k \geq 0}\left(\begin{array}{c}
C+k-1 \\
k
\end{array}\right) z^{k}=\frac{1}{(1-z)^{C}} .
$$

Since $T(\rho) \leq \hat{B}(\rho) \leq 1 / \sqrt{2 n}$ (a tautology cannot be a single leaf) and $\frac{C}{(1-z)^{C+1}}-C=O(z)$, we obtain

$$
\mu_{n}\left(E^{\geq 2}\left(\mathcal{M}_{f}\right)\right)=\mathcal{O}\left(\frac{1}{n^{L(f)+1 / 2}}\right) .
$$

By 13 the same calculations yield the lower bound.

\subsection{Irreducible trees.}

Definition 9.3. Let $t$ be a tree computing $f$. If $t$ cannot be obtained by a tautology expansion of a smaller tree computing $f$, then $t$ is called irreducible. We denote by $\mathcal{I}_{f}$ the set of irreducible trees of $f$ which are not minimal trees of $f$.

Take a tree computing $f$ and simplify it according to tautology expansions until it is irreducible. The simplified tree is either in $\mathcal{M}_{f}$ or in $\mathcal{I}_{f}$. Thus

$$
\mathbb{P}_{n}(f) \leq \mu_{n}\left(E^{*}\left(\mathcal{M}_{f}\right)\right)+\mu_{n}\left(E^{*}\left(\mathcal{I}_{f}\right)\right) .
$$

Proposition 9.4. We have the following asymptotic result: $\mu_{n}\left(E^{*}\left(I_{f}\right)\right)=o\left(1 / n^{L(f)}\right)$.

To prove this proposition, we have to understand better the shape of an irreducible tree of $f$. Let $t$ be such a tree. Let us "simplify" the tree as follows:

- assign all leaves of $t$ which are labelled by inessential variables ${ }^{2}$ of $f$ to True, and then

- simplify the tree as follows: As soon as a leaf is assigned to True (resp. False) and its parent is $\wedge$ (resp. $\vee$ ), we cut the leaf. If its parent is $\vee($ resp. $\wedge)$, we cut the subtree rooted at this $\vee($ resp. $\wedge)$, i.e. at the parent.

The obtained tree, denoted by $t^{\star}$ contains no inessential variable and still computes $f$. It possibly has internal nodes with a single child (called unary nodes), and connectives labelling some leaves (where $\vee$-leaves compute False and $\wedge$-leaves compute True, the other leaves being labelled by essential variables of $f$. But, since this tree computes $f$, it can be seen that its size cannot be smaller than $L(f)$. Indeed, a tree with unary nodes and leaves labelled by connectives can be simplified such that we obtain a proper and/or tree that still computes $f$, i.e. with at least $L(f)$ nodes, and this simplification process reduces the number of nodes. The tree $t^{\star}$ belongs to the following family of trees:

\footnotetext{
${ }^{2} \mathrm{~A}$ variable $x$ is an inessential variable of $f$ if the restriction of $f$ on the subset $\{x=$ True $\}$ is equal to its restriction on the subset $\{x=$ False $\}$.
} 
Definition 9.5. Let us denote by $\mathcal{S}$ the set of trees with internal nodes labelled by $\wedge$ and $\vee$ in a stratified way and with leaves labelled by essential variables of $f$ (or their negations) or $\wedge$ and $\vee$ (again in compliance with the stratification), in which internal nodes can have one child or more. We denote by $S_{\ell}$ the number of such trees of size $\ell$ (the size being the total number of nodes of the tree).

Remark: The number $S_{\ell}$ only depends on $\gamma$, the number of essential variables of $f$, and on $\ell$.

Note that during the simplification process, we have cut either leaves or non-leaf subtrees. We will prove in the following lemmas that the family of trees such that the simplification process cuts no non-leaf tree and the family of trees in which we have cut "many" single leaves is negligible in comparison with the family of trees computing $f$ (the limiting ratio of which is at least of order $1 / n^{L(f)}$ ).

Lemma 9.6. Let $\Gamma \subseteq\left\{x_{1}, \ldots, x_{n}\right\}$ be of cardinality $\gamma$ and set $Y=\left\{x_{1}, \ldots, x_{n}\right\} \backslash \Gamma$. Moreover, let $\mathcal{N}_{\gamma}$ be the family of trees such that no node labelled by $\vee($ resp. $\wedge)$ has a leaf labelled by a positive (resp. negated) variable from $Y$ as a child. Then $\mu_{n}\left(\mathcal{N}_{\gamma}\right)=0$, when $n$ is large enough.

Note that the family $\mathcal{N}_{\gamma}$ contains the family of trees computing a function $f$ having $\Gamma$ as its set of essential variables and such that the simplification process only cuts leaves and no non-leaf tree.

Proof. The family $\mathcal{N}_{\gamma}$ has the same limiting ratio as associative trees in which leaves are labelled by literals from a set of cardinality $2 n-(n-\gamma)=n+\gamma$. Therefore, the singularity $\nu_{n}$ of the generating function of this family is of squareroot type and satisfies $\nu_{n} \sim 1 / n+\gamma$ and is thus strictly larger than $\rho_{n} \sim 1 / 2 n$ for large enough $n$. This implies the assertion.

The following lemma ensures that we have cut only a few "single" leaves:

Lemma 9.7. Let $\ell$ be an integer and $\Gamma \subseteq\left\{x_{1}, \ldots, x_{n}\right\}$ be of cardinality $\gamma$. Set $Y=\left\{x_{1}, \ldots, x_{n}\right\} \backslash \Gamma$ and let us denote by $\mathcal{N}_{\ell}$ the family of trees with at least $\ell$ leaves labelled by variables from $Y$ (or their negations) such that none of these leaves has an ancestor labelled by $\vee($ resp. $\wedge)$ which has a child being a leaf labelled by a positive (resp. negated) variable from $Y$. Then $\mu_{n}\left(\mathcal{N}_{\ell}\right)=o\left(1 / n^{\ell+1}\right)$.

Note that the family $\mathcal{N}_{\ell}$ contains the family of trees computing a function $f$ having $\Gamma$ as its set of essential variables and in which the simplification process cuts at least $\ell$ single leaves.

Proof. Let us consider the family of trees obtained as follows (see Figure 2):

(1) Take a rooted unlabelled tree $t_{0}$ having $\ell$ leaves and no nodes of arity 1 , with $\ell+1 \leq\left|t_{0}\right| \leq 2 \ell-2$;

(2) add to each internal node some subtrees (with internal nodes unlabelled and leaves labelled by literals) which are not single leaves labelled by a variable from $Y$ or its negation,

(3) replace each edge by a sequence of internal (unlabelled) nodes with subtrees; (with internal nodes unlabelled and leaves labelled by literals) attached to them which are not single leaves labelled by a positive (resp. negated) variable from $Y$ if their parent is $\vee($ resp. $\wedge$ );

(4) choose a label $(\wedge$ or $\vee$ ) for the root and deduce the labels of all internal nodes, knowing that a node and its child cannot have the same label (stratification property);

(5) finally, replace each leaf of $t_{0}$ by a tree rooted by $\wedge$ (resp. $\vee$ according to the stratification) with at least one literal from $Y$ (resp. from the negations of $Y$ ) and no literal from the negations of $Y$ (resp. from $Y$ ) on the first level.

The obtained family contains $\mathcal{N}_{\ell}$ and its generating function is given by

$$
F(z)=2 C_{\ell} \sum_{r=\ell}^{2 \ell-3} z^{r+1-\ell}(z X(z))^{\ell} V(z)^{r} W(z)^{2 r+1-\ell},
$$

where

- the index $r$ in the summation represents the number of edges of the tree $t_{0}$ chosen in the construction;

- the factor $C_{\ell}$ is the number of choices for this $t_{0}$ and the factor 2 for its root label;

- the factor $z^{r+1-\ell}$ marks the internal nodes of $t_{0}$;

- the function

$$
z X(z)=\frac{(n-\gamma) z^{2}}{[1-(\hat{A}(z)-2(n-\gamma) z)][1-(\hat{A}(z)-(n-\gamma) z))]}-(n-\gamma) z^{2}
$$




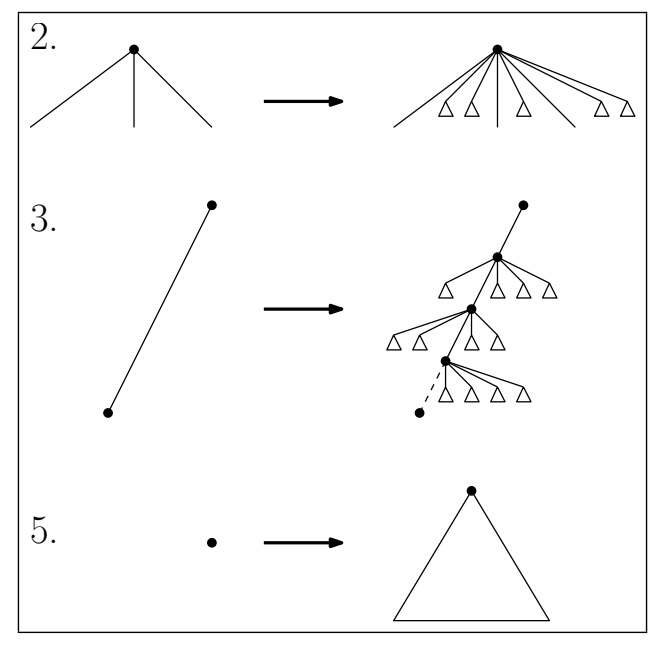

Figure 2. Proof of Lemma 9.7 .

is the generating function of the set of trees rooted by $\wedge$ with at least one literal from $Y$ and no literal from the negations of $Y$ on the first level;

- the function

$$
V(z)=\frac{1}{1-z\left(\left(\frac{1}{1-(\hat{A}(z)-(n-\gamma) z)}\right)^{2}-1\right)}
$$

is the generating function of the sequences of internal nodes that replace the edges of $t_{0}$ : It is the generating function of sequences (possibly empty) of one internal node marked by $z$ and two sequences (one on the left and one on the right of the existing edge - that cannot be both empty) of subtrees being different from a leaf labelled by a positive variable of $Y$; and

- the function

$$
W(z)=\frac{1}{1-(\hat{A}-(n-\gamma) z)}
$$

is the generating function of the sequences of trees which are different from a single leaf with a label from $Y$. Note that such sequences are attached in the 3 rd step and they can be placed left from every edge in $t_{0}$ ( $r$ choices) or to the right of the rightmost child of any internal node of $t_{0}(r+1-\ell$ choices).

To estimate the limiting ratio of this family, observe that the singularity of $F$ is $\rho$ and that it is a squareroot singularity. Therefore, the limiting ratio of this family can be computed by Lemma 4.2 i.e. by $\lim _{z \rightarrow \rho} \frac{F^{\prime}(z)}{A^{\prime}(z)}$. To compute this limiting ratio, let us note that $\lim _{z \rightarrow \rho} \frac{1}{A^{\prime}(z)}=0$ such that many terms in $F^{\prime}(z) / A^{\prime}(z)$ can be neglected (cf. last paragraph in the proof of Proposition 4.3). We obtain:

$$
\begin{aligned}
\lim _{z \rightarrow \rho} \frac{F^{\prime}(z)}{A^{\prime}(z)}=2 C_{\ell} \sum_{r=\ell+1}^{2 \ell-2} \rho^{r+1}( & \ell(\rho)^{\ell-1} V(\rho)^{r} W(\rho)^{2 r+1-\ell} \lim _{z \rightarrow \rho} \frac{X^{\prime}(z)}{A^{\prime}(z)} \\
& +r X(\rho)^{\ell} V(\rho)^{r-1} W(\rho)^{2 r+1-\ell} \lim _{z \rightarrow \rho} \frac{V^{\prime}(z)}{A^{\prime}(z)} \\
& \left.+(2 r+1-\ell) X(\rho)^{\ell} V(\rho)^{r} W(\rho)^{2 r-\ell} \lim _{z \rightarrow \rho} \frac{W^{\prime}(z)}{A^{\prime}(z)}\right)
\end{aligned}
$$

Recall that $\rho \sim 1 / 2 n$ and $\hat{A}(\rho) \sim 1-1 / \sqrt{2 n}$, as $n \rightarrow \infty$, and hence $X(\rho) \sim 1 / 3, V(\rho) \sim 1$ and $W(\rho) \sim 2$, as $n \rightarrow \infty$. Moreover,

$$
\lim _{z \rightarrow \rho} \frac{W^{\prime}(z)}{A^{\prime}(z)}=\lim _{z \rightarrow \rho} \frac{\hat{A}^{\prime}(z)-(n-\gamma)}{A^{\prime}(z)} \frac{1}{(1-(\hat{A}(\rho)-(n-\gamma) \rho))^{2}}=\frac{1}{2(1-(\hat{A}(\rho)-(n-\gamma) \rho))^{2}},
$$


because $\lim _{z \rightarrow \rho} \frac{\hat{A}^{\prime}(z)}{A^{\prime}(z)}=\frac{1}{2}$ and $\lim _{z \rightarrow \rho} \frac{n-\gamma}{A^{\prime}(z)}=0$. Thus, $\lim _{n \rightarrow \infty} \lim _{z \rightarrow \rho} \frac{W^{\prime}(z)}{A^{\prime}(z)}=2$. Using similar arguments, we can prove that

All these relations imply

$$
\lim _{n \rightarrow \infty} \lim _{z \rightarrow \rho} \frac{V^{\prime}(z)}{A^{\prime}(z)}=8 \quad \text { and } \quad \lim _{z \rightarrow \rho} \frac{X^{\prime}(z)}{A^{\prime}(z)} \sim \frac{8 n}{9}, \text { as } n \rightarrow \infty .
$$

where $\kappa$ is a positive constant.

$$
\lim _{z \rightarrow \rho} \frac{F^{\prime}(z)}{A^{\prime}(z)} \sim \frac{\kappa}{n^{\ell+1}}, \text { as } n \rightarrow \infty
$$

We are now ready to prove Proposition 9.4. The two previous lemmas allow us to consider only irreducible trees in which the simplification process cuts at least one non-leaf tree and fewer than $L(f)$ single leaves. Let us denote by $\mathcal{I}_{1}$ the set of irreducible trees for which the simplified tree $t^{\star}$ has size $L(f)$, and by $\mathcal{I}_{2}$ the set of irreducible trees $t$ of $f$ such that $t^{\star}$ has size at least $L(f)+1$.

Lemma 9.8. We have the following asymptotic result: $E^{*}\left(\mathcal{I}_{1}\right)=o\left(1 / n^{L(f)}\right)$.

Proof. Let $t \in \mathcal{I}_{1}$. To obtain $t^{\star}$, we have cut subtrees of $t$ and we can assume that we have cut at least one large subtree and at most $L(f)$ single leaves. Assume first that we have cut only one non-leaf subtree rooted at a node $\nu$ during the algorithm. Then, either this tree contains an essential variable on its first-level leaves, or it belongs to $\bigcup_{k, \ell>0} \bigcup_{r=0}^{\ell-1} \mathcal{M}_{k, \ell, r}^{\Gamma_{f}}$, where $\Gamma_{f}$ is the set of essential variables of $f$. Otherwise, we could find an assignment of inessential variables such that we can cut the father of $\nu$ without changing the function computed by the tree $t$. This new assignment of inessential variables leads to a different simplification of the tree $t$ that will cut at least the single leaves that were cut before, plus the larger subtree and its father: We thus obtain a tree of size less than $L(f)$ that computes $f$, which is impossible.

Any tree of $\mathcal{I}_{1}$ is obtained by expanding a tree $s$ of $\mathcal{S}_{L(f)}$ as follows:

- choose an integer $q \geq 1$ ( $q$ represents the number of large trees that were cut during the process described beforehand: $q \geq 1$ holds because of the remark before Lemma 9.8,

- if $q=1$, plug a tree from $\left(\bigcup_{k, \ell \geq 0} \bigcup_{r=0}^{\ell-1} \mathcal{M}_{k, \ell, r}^{\Gamma_{f}}\right) \cup \mathcal{A}_{\Gamma_{f}}$ at a node of $s$ and at most $L(f)$ inessential leaves at other nodes of $s$ (where $\mathcal{A}_{\Gamma_{f}}$ is the set of trees containing at least one first-level leaf labelled by an essential variable of $f, c f$. Proposition 4.4,

- else plug $q \geq 2$ non-leaf subtrees and at most $L(f)$ inessential leaves at nodes of $s$.

We are interested in expansions of trees from $\mathcal{I}_{1}$. In fact, since we do not impose any restrictions on the trees we plug at nodes of $s$, we can consider only expansions in the nodes of $s$, since expansions in the plugged trees are then already counted.

The generating function of trees obtained by successive expansions of trees from $\mathcal{I}_{1}$, denoted by $I_{1}(z)$ thus satisfies:

$$
\begin{aligned}
I_{1}(z) \prec S_{L(f)} z^{L(f)} \sum_{k \geq 0}\left(\begin{array}{c}
3 L(f)+1+k \\
L(f)+1, k, 2 L(f)
\end{array}\right) M(z)(2(n-\gamma) z)^{L(f)} \hat{B}(z)^{k} \\
\left.\quad+\sum_{q \geq 2}\left(\begin{array}{c}
3 L(f)+q+k \\
L(f)+q, k, 2 L(f)
\end{array}\right) \hat{B}(z)^{q+k}(2(n-\gamma) z)^{L(f)}\right),
\end{aligned}
$$

where $k$ counts the number of successive expansions done into the irreducible tree, and where $M(z)$ is the generating function of $\left(\bigcup_{k, \ell \geq 0} \bigcup_{r=0}^{\ell-1} \mathcal{M}_{k, \ell, r}^{\Gamma_{f}}\right) \cup \mathcal{A}_{\gamma}$. The multinomial coefficient represents the number of choices for the places where we plug trees in $s$ and where we then do the expansions (the orders of the "pluggings" and of the expansions do not matter, but expansions are done after the "pluggings").

The coefficient $\left(\begin{array}{c}3 L(f)+1+k \\ L(f)+1, k, 2 L(f)\end{array}\right)$ counts the different ways to plug one tree from $\left(\bigcup_{k, \ell \geq 0} \bigcup_{r=0}^{\ell-1} \mathcal{M}_{k, \ell, r}^{\Gamma_{f}}\right) \cup \mathcal{A}_{\gamma}$, $L(f)$ inessential leaves and $k \hat{B}$-expansions into $s \in \mathcal{S}_{L(f)}$. Let us start from $s$, the first plugging can be done at the left of each edge of $s$, or at the right of every rightmost edge, or at every leaf. Note that $s \in \mathcal{S}_{L(f)}$ has at most $L(f)$ leaves and $L(f)$ edges. The number of different places for the first plugging is thus at most $3 L(f)$. The second plugging can then be made in $3 L(f)+1$ different places, and so on, and so forth. The fact that we first do the $L(f)+1$ plugging and then the $k$ expansions gives the multinomial coefficient. The second binomial coefficient is given by the same reasonning with $L(f)+q$ pluggings and $k$ expansions. 
Thanks to Proposition 4.4 and Section 5 , we know that the limiting ratio of $\left(\bigcup_{k, \ell \geq 0} \bigcup_{r=0}^{\ell-1} \mathcal{M}_{k, \ell, r}^{\Gamma_{f}}\right) \cup \mathcal{A}_{\Gamma_{f}}$ has order $\mathcal{O}\left(\frac{1}{\sqrt{n}}\right)$ and that $M(\rho) \leq \hat{B}(\rho) \sim \frac{1}{\sqrt{2 n}}$. Thus, when $n$ tends to infinity,

$$
\begin{aligned}
\mu_{n}\left(E^{*}\left(\mathcal{I}_{1}\right)\right) \leq S_{L(f)} \rho^{L(f)} \hat{B}(\rho) \sum_{k \geq 0} & \left(\begin{array}{c}
3 L(f)+1+k \\
L(f)+1, k, 2 L(f)
\end{array}\right)\left(\hat{B}(\rho)^{k}+\frac{k}{2} \hat{B}(\rho)^{k-1}\right) \\
& \left.+\sum_{q \geq 2}\left(\begin{array}{c}
3 L(f)+q+k \\
L(f)+q, k, 2 L(f)
\end{array}\right) \frac{q+k}{2} \hat{B}(\rho)^{q+k-2}\right) .
\end{aligned}
$$

The first factor $S_{L(f)} \rho^{L(f)} \hat{B}(\rho)$ behaves as $\frac{1}{n^{L(f)+1 / 2}}$ when $n$ tends to infinity. Let us prove that the second term of the sum behaves as $\mathcal{O}(1)$ when $n$ tends to infinity. Let us first focus on

$$
\begin{aligned}
\sum_{k \geq 0}\left(\begin{array}{c}
3 L(f)+1+k \\
L(f)+1, k, 2 L(f)
\end{array}\right) \hat{B}(\rho)^{k} & =\frac{(3 L(f)+1) !}{(L(f)+1) !(2 L(f)) !} \sum_{k \geq 0}\left(\begin{array}{c}
3 L(f)+1+k \\
k
\end{array}\right) \hat{B}(\rho)^{k} \\
& =\frac{(2 L(f)+2) !}{(L(f)+1) !(2 L(f)) !} \frac{1}{(1-\hat{B}(\rho))^{3 L(f)+2}}
\end{aligned}
$$

in view of (14). Very similar calculations lead to

$$
\sum_{k \geq 0}\left(\begin{array}{c}
3 L(f)+1+k \\
L(f)+1, k
\end{array}\right) \frac{k}{2} \hat{B}(\rho)^{k-1}=\frac{(3 L(f)+1) !}{2(3 L(f)+1) !(2 L(f) !)} \frac{3 L(f)+2}{(1-\hat{B})^{3 L(f)+3}} .
$$

Moreover, using (14) again,

$$
\begin{aligned}
& \sum_{k \geq 0} \sum_{q \geq 2}\left(\begin{array}{c}
3 L(f)+q+k \\
L(f)+q, k, 2 L(f)
\end{array}\right) \frac{q}{2} \hat{B}(\rho)^{q+k-2} \\
& =\sum_{q \geq 2} \frac{(3 L(f)+q) !}{(L(f)+q) !(2 L(f)) !} \frac{q}{2} \hat{B}(\rho)^{q-2} \sum_{k \geq 0}\left(\begin{array}{c}
3 L(f)+q+k \\
k
\end{array}\right) \hat{B}(\rho)^{k} \\
& =\frac{1}{2(1-\hat{B}(\rho))^{3 L(f)+3}} \sum_{q \geq 0}\left(\begin{array}{c}
3 L(f)+q+2 \\
L(f)+q+2
\end{array}\right)(q+2)\left(\frac{\hat{B}(\rho)}{(1-\hat{B}(\rho))}\right)^{q} \\
& =\frac{1}{2(1-\hat{B}(\rho))^{3 L(f)+3}} \sum_{q \geq 0}\left[\prod_{j=2}^{L(f)+2}\left(\frac{2 L(f)+q+j}{q+j}\right)\right]\left(\begin{array}{c}
2 L(f)+q+1 \\
q+1
\end{array}\right)(q+2)\left(\frac{\hat{B}(\rho)}{1-\hat{B}(\rho)}\right)^{q} \\
& \leq \frac{(1+2 L(f))^{L(f)+1}}{2(1-\hat{B}(\rho))^{3 L(f)+3}} \sum_{q \geq 0}\left(\begin{array}{c}
2 L(f)+q+1 \\
q+1
\end{array}\right)(q+2)\left(\frac{\hat{B}(\rho)}{1-\hat{B}(\rho)}\right)^{q} \\
& =\frac{(1+2 L(f))^{L(f)+1}}{2(1-\hat{B}(\rho))^{3 L(f)+3}}\left(\frac{2 L(f)+1}{\left(1-\frac{\hat{B}(\rho)}{1-\hat{B}(\rho)}\right)^{2 L(f)+2}}+\frac{1}{\left(1-\frac{\hat{B}(\rho)}{1-\hat{B}(\rho)}\right)^{2 L(f)+1}}\right),
\end{aligned}
$$

since $\frac{\hat{B}(\rho)}{1-\hat{B}(\rho)}$ is smaller than 1 for large enough $n$. Similar calculations can be done for the last term of the sum (16), and we eventually get

$$
\mu_{n}\left(E^{*}\left(\mathcal{I}_{1}\right)\right)=\mathcal{O}\left(\frac{1}{n^{L(f)+1 / 2}}\right)
$$

Lemma 9.9. We have the following asymptotic result: $E^{*}\left(\mathcal{I}_{2}\right)=o\left(1 / n^{L(f)}\right)$.

Proof. The generating function $I_{2}(z)$ of trees obtained by successive expansions of a tree from $\mathcal{I}_{2}$ satisfies

$$
I_{2}(z) \leq \sum_{\ell \geq L(f)+1} S_{\ell} z^{\ell} \sum_{k \geq 0} \sum_{q \geq 0}\left(\begin{array}{c}
2 \ell+L(f)+q+k \\
L(f)+q, k, 2 \ell
\end{array}\right) \hat{B}(z)^{k+q}(2(n-\gamma) z)^{L(f)}
$$


where $S_{\ell}$ is the number of trees, where $\ell$ is the size of the seminal tree in $\mathcal{I}_{2}$, and where the multinomial coefficient counts the numbers of different ways to plug first $L(f)$ leaves and $q$ non-leaf trees and then $k$ expansions in this seminal tree. Thus, calculations in the same vein as those done in the proof of Lemma 9.8 yield

$$
\begin{aligned}
\mu_{n}\left(E^{*}\left(\mathcal{I}_{2}\right)\right) & \leq \sum_{\ell \geq L(f)+1} S_{\ell} \rho^{\ell} \sum_{k \geq 0} \sum_{q \geq 0}\left(\begin{array}{c}
2 \ell+L(f)+q+k \\
L(f)+q, k, 2 \ell
\end{array}\right) \frac{k+q}{2} \frac{1}{\sqrt{n}^{k+q-1}} \\
& =\mathcal{O}\left(\frac{1}{n^{L(f)+1}}\right) .
\end{aligned}
$$

Lemmas 9.8 and 9.9 directly induce Proposition 9.4 and we are now able to complete the proof of Theorem 2.4

Proof of Theorem 2.4. The probabilities of the set of tautologies and that of literals are treated in Sections 6 and 8 respectively. We now have to prove that for all Boolean function $f, \mathbb{P}_{n}(f)=\Theta\left(1 / n^{L(f)}\right)$. In view of Proposition 9.2 we have $\mathbb{P}_{n}(f)=\Omega\left(1 / n^{L(f)}\right)$, and 15 together with Proposition 9.4 gives $\mathbb{P}_{n}(f)=\mathcal{O}\left(1 / n^{L(f)}\right)$ which completes the proof of Theorem 2.4 .

\section{CONClusion AND FURTher WORK}

This article presents the first attempt to discuss the size definition in quantitative logics: while the formula size is commonly used in the literature, we believe that in the case of non binary trees, the tree size considered in this article is at least as natural.

We proved that this change of size/complexity notion does not affect the first order behaviour of the distribution induced on the set of Boolean functions by the uniform distribution on and/or trees of a given (large) size and labelled on $n$ variables. However, we have also exhibited how the large typical and/or tree has a very different shape when changing the size notion. Trees having more leaves are more and more likely as the number $n$ of variables increases. This change of typical shape has forced us to develop original, more intricate proofs for this new model.

Note that our main result only gives a $\Theta$-asymptotic whereas for the formula size associative trees model (see [15]) a stronger equivalent result can be obtained. We strongly believe that the following holds: for all Boolean function, there exists a constant $\lambda_{f}>0$ such that, when $n \rightarrow+\infty$,

$$
\mathbb{P}_{n}(f) \sim \frac{\lambda_{f}}{n^{L(f)}},
$$

but this requires a much deeper understanding of the structure of tautologies and certainly even more technical calculations.

Considering non binary tree is a way to take into account the associativity of the logical connectives $\wedge$ and $\vee$. Note that there is no reason - except technicality - justifying that we consider plane trees instead of non plane trees while the connectives $\wedge$ and $\vee$ are commutative. Non plane associative trees have been studied in the formula size model (see [15), and we believe that the same could be done for the tree size model, although once again, the technical level of the computations would considerably increase since in non plane models, the generating functions are not known explicitly.

\section{REFERENCES}

[1] R. B. Boppana. Amplification of probabilistic Boolean formulas. In Proceedings of the 26th Annual IEEE Symposium on Foundations of Computer Science, pages 20-29, 1985.

[2] A. Brodsky and N. Pippenger. The Boolean functions computed by random Boolean formulas or how to grow the right function. Random Structures and Algorithms, 27:490-519, 2005.

[3] B. Chauvin, P. Flajolet, D. Gardy, and B. Gittenberger. And/Or trees revisited. Combinatorics, Probability and Computing, 13(4-5):475-497, July-September 2004.

[4] M. Drmota. Systems of functional equations. Random Structures and Algorithms, 10(1-2):103-124, 1997.

[5] M. Drmota. Random trees. Springer, Vienna-New York, 2009.

[6] P. Flajolet, D. Gardy, and L. Thimonier. Birthday Paradox, Coupon Collectors, Caching Algorithms and Self-Organizing Search. D. A. Math., 39(3):207-229, 1992.

[7] P. Flajolet and A. M. Odlyzko. Singularity analysis of generating functions. In SIAM J. Discrete Math., 3:216-240, 1990.

[8] P. Flajolet and R. Sedgewick. Analytic Combinatorics. Cambridge U.P., Cambridge, 2009.

[9] H. Fournier, D. Gardy, and A. Genitrini. Balanced And/Or trees and linear threshold functions. In Proceedings of the 5th SIAM Workshop on Analytic and Combinatorics, pages 51-57, 2009.

[10] H. Fournier, D. Gardy, A. Genitrini, and B. Gittenberger. Complexity and limiting ratio of Boolean functions over implication. In MFCS, pages 347-362, 2008. 
[11] H. Fournier, D. Gardy, A. Genitrini, and B. Gittenberger. The fraction of large random trees representing a given boolean function in implicational logic. Random Structures and Algorithms, 40(3):317-349, 2012.

[12] D. Gardy. Random Boolean expressions. In Colloquium on Computational Logic and Applications, volume AF, pages 1-36. DMTCS Proceedings, 2006.

[13] A. Genitrini and B. Gittenberger. No Shannon effect on probability distributions on Boolean functions induced by Boolean expressions. In Proc. of Analysis of Algorithms, pages 303-316, Wien, Austria, July 2010. DMTCS Proceedings AM.

[14] A. Genitrini, B. Gittenberger, V. Kraus, and C. Mailler. Probabilities of boolean functions given by random implicational formulas. Electronic Journal of Combinatorics, 19(2):P37, 20 pages, (electronic), 2012.

[15] A. Genitrini, B. Gittenberger, V. Kraus, and C. Mailler. Associative and commutative tree representations for boolean functions. Theoretical Computer Science, 570(0):70-101, 2015.

[16] A. Genitrini, B. Gittenberger, and C. Mailler. No Shannon effect induced by And/Or trees. In 25th International Meeting on Probabilistic, Combinatorial and Asymptotic Methods for the Analysis of Algorithms, pages 109-120, Paris, France, June 2014.

[17] R. L. Graham, M. Grötschel, and L. Lovász, editors. Handbook of Combinatorics (Vol. 2). MIT Press, Cambridge, MA, USA, 1995.

[18] A. Gupta and S. Mahajan. Using amplification to compute majority with small majority gates. Computational Complexity, 6(1):4663, 1997.

[19] S. Jukna. On graph complexity. Combinatorics, Probability \& Computing, 15(6):855-876, 2006.

[20] S. Jukna. Boolean Function Complexity - Advances and Frontiers, volume 27 of Algorithms and combinatorics. Springer, 2012.

[21] Jakub Kozik. Subcritical pattern languages for and/or trees. In Fifth Colloquium on Mathematics and Computer Science, Discrete Math. Theor. Comput. Sci. Proc., AI, pages 437-448. Assoc. Discrete Math. Theor. Comput. Sci., Nancy, 2008.

[22] S. P. Lalley. Finite range random walk on free groups and homogeneous trees. The Annals of Probability, $21,1993$.

[23] H. Lefmann and P. Savický. Some typical properties of large And/Or Boolean formulas. Random Structures and Algorithms, 10:337-351, 1997.

[24] O. B. Lupanov. Complexity of formula realization of functions of logical algebra. Problemy Kibernetiki, 3:61-80, 1960. English translation: Problems of Cybernetics, Pergamon Press, 3:782-811, 1962.

[25] O. B. Lupanov. On the realization of functions of logical algebra by formulae of finite classes (formulae of limited depth) in the basis $\wedge, \vee, \neg$. Problemy Kibernetiki, 6:5-14, 1961. English translation: Problems of Cybernetics, Pergamon Press, 6:1-14, 1965.

[26] J. B. Paris, A. Vencovská, and G. M. Wilmers. A natural prior probability distribution derived from the propositional calculus. Ann. Pure Appl. Logic, 70(3):243-285, 1994.

[27] A. A. Razborov. Lower bounds for propositional proofs and independence results in bounded arithmetic. In Automata, Languages and Programming, 23rd International Colloquium, ICALP96, Paderborn, Germany, 8-12 July 1996, Proceedings, pages 48-62, 1996.

[28] J. Riordan and C. E. Shannon. The number of two terminal series-parallel networks. Journal of Mathematics and Physics, 21:83-93, 1942.

[29] P. Savický. Random Boolean formulas representing any Boolean function with asymptotically equal probability. Discrete Mathematics, 83:95-103, 1990.

[30] C. E. Shannon. Communication theory of secrecy systems. Bell System Tech. J., 28:656-715, 1949.

[31] L. Valiant. Short monotone formulae for the majority function. Journal of Algorithms, 5:363-366, 1984.

[32] A. R. Woods. Coloring rules for finite trees, and probabilities of monadic second order sentences. Random Structures and Algorithms, 10(4):453-485, 1997

[33] A. C. Yao. Separating the polynomial-time hierarchy by oracles. In 26th Annual Symposium on Foundations of Computer Science, pages $1-10,1985$. 